\title{
Partonic quasidistributions in two-dimensional QCD
}

\author{
Yu Jia, ${ }^{1,2, *}$ Shuangran Liang, ${ }^{1,2, \dagger}$ Xiaonu Xiong, ${ }^{3, *}$ and Rui $\mathrm{Yu}^{1,2, \S}$ \\ ${ }^{1}$ Institute of High Energy Physics, Chinese Academy of Sciences, Beijing 100049, China \\ ${ }^{2}$ School of Physics, University of Chinese Academy of Sciences, Beijing 100049, China \\ ${ }^{3}$ Institute for Advanced Simulation, Institut für Kernphysik and Jülich Center for Hadron Physics, \\ Forschungszentrum Jülich, D-52425 Jülich, Germany
}

(Received 1 May 2018; published 10 September 2018)

\begin{abstract}
As a sequel to our preceding work [Y. Jia et al. J. High Energy Phys. 11 (2017) 151], we carry out a comprehensive comparative study between the quasiparton distribution functions (PDFs), distribution amplitudes (DAs), and their light-cone counterparts for various flavor-neutral mesons in the context of the 't Hooft model, that is, the two-dimensional QCD in the large- $N$ limit. In contrast to the original derivation via diagrammatic techniques exemplified by Dyson-Schwinger and Bethe-Salpeter equations, here we employ the Hamiltonian operator approach to reconstruct the celebrated 't Hooft equation in light-front quantization, and Bars-Green equations in equal-time quantization. The novelty of our derivation is to employ the soft momentum cutoff as the IR regulator. As a virtue of this operator approach, the functional form of the quasidistributions can be transparently built out of the Bars-Green wave functions and the Bogoliubov angle with the aid of bosonization technique. Equipped with various bound-state wave functions numerically inferred by Jia et al. [J. High Energy Phys. 11 (2017) 151], we then investigate how rapidly the quasidistributions approach their light-cone counterparts with the increasing meson momentum. We observe that the light mesons' quasidistributions approach the light-cone distributions at a slower pace than the heavy quarkonia. Curiously, lattice simulations of quasidistributions in fourdimensional QCD also discover this feature. Furthermore, we also compute the partonic light-cone PDF and quasi-PDF to one-loop order in perturbation theory, again employing the momentum cutoff as the IR regulator. We explicitly verify one of the backbones underlying the large momentum effective field theory (LaMET), namely, both quasi-PDFs and light-cone PDFs in $\mathrm{QCD}_{2}$ indeed possess the same IR behavior at leading order in $1 / P^{z}$.
\end{abstract}

DOI: 10.1103/PhysRevD.98.054011

\section{INTRODUCTION}

Parton distributions functions (PDFs) and distribution amplitudes (DAs) encapsulate the nonperturbative structures of quarks and gluons inside a hadron. Parton distributions are the key ingredient for making predictions for any hard process in the high-energy hadron collision experiments. Undoubtedly, the most promising approach of calculating the parton distributions from the first principle of QCD is lattice simulation. Nevertheless, due to their intrinsic Minkowski nature, it is very difficult to directly deduce the parton distributions as functions of $x$ on

\footnotetext{
*jiay@ihep.ac.cn

liangsr@ihep.ac.cn

*x.xiong@fz-juelich.de

§yurui@ihep.ac.cn
}

Published by the American Physical Society under the terms of the Creative Commons Attribution 4.0 International license. Further distribution of this work must maintain attribution to the author(s) and the published article's title, journal citation, and DOI. Funded by SCOAP ${ }^{3}$.
Euclidean lattice. Until recently, only the first few Mellin moments of parton distributions were accessible to lattice study [1-5].

A breakthrough occurred several years ago, exemplified by the introduction of quasidistributions and the large momentum effective field theory (LaMET) [6,7]. This novel approach principally paves the way for directly calculating the $x$ dependence of parton distributions on Euclidean lattice. Reference [8] explicitly shows that the infrared structures of the quark quasi-PDF and light-cone PDF are identical at one-loop level; consequently, the matching factor linking these two sets of PDFs was derived to this perturbative order. The factorization theorem conjectured in [6] that links the quasi-PDF and ordinary PDF is later proved to all orders in $\alpha_{s}$ [9]. The renormalization of quasiquark PDFs to all orders in $\alpha_{s}$ is addressed in Refs. [10,11]. The nonperturbative matching program has also been discussed $[12,13]$. A plethora of exploratory lattice simulations of quark quasi-PDFs, DAs have become available recently [14-23]. Moreover, studies have appeared based on lattice perturbation theory for quasi-PDFs [24-26], and some 
improvement of quasi-PDFs is suggested in Refs. [27-29]. We also notice that one-loop matching for the gluon quasiPDF has also recently been explored [30].

Solving the realistic four-dimensional QCD is a notoriously difficult mission. Conceivably, there is still a long way to proceed before obtaining the phenomenologically competitive parton distributions from the angle of lattice simulations. In the meanwhile, it may also look attractive if we can learn something useful about partonic quasidistributions from much more tractable model field theories. To date, most solvable field theories live in 1+1-dimensional spacetime. In this paper, we will utilize the two-dimensional QCD (hereafter, abbreviated $\mathrm{QCD}_{2}$ ) as a specific toy model, to unravel various aspects of (quasi)partonic distributions. Curiously, some qualitative features observed in this work, especially when regarding the behavior of quasidistributions under boost, are not unique to $\mathrm{QCD}_{2}$ only but instead are also captured by realistic $\mathrm{QCD}_{4}$.

The $1 / N$ expansion has historically served a powerful nonperturbative tool of QCD since the theory becomes considerably simpler in the large- $N$ limit [31-33]. Some essential nonperturbative features of strong interactions are impressively captured in this limit. In a similar vein, $\mathrm{QCD}_{2}$ in the large- $N$ limit, often referred to as the 't Hooft model [34], turns out to be an exactly solvable model. Via diagramatically based Dyson-Schwinger and BetheSalpeter methods, 't Hooft was able to resum the planar diagrams to arrive at the bound state equation in the context of light-cone quantization and light-cone gauge. The resulting bound state wave function can be readily interpreted as the light-cone wave function (LCWFs). Lightcone correlations can, thus, be naturally constructed out of the 't Hooft wave function. For instance, PDF and GPD in $\mathrm{QCD}_{2}$ have been studied long ago by Burkardt [35].

The motif of this work is to carefully investigate the nature and characteristics of quasi-PDFs and DAs for various flavor-neutral mesons in the 't Hooft model. To this purpose, a reformulation of $\mathrm{QCD}_{2}$ in the equal-time quantization, looks much more appropriate. A theoretical foundation along this line was first laid down by Bars and Green in 1978 [36]. We will illustrate how to express the quasidistributions in terms of more fundamental building blocks in 't Hooft model, a pair of bound-state wave functions first introduced in [36]. We will be particularly interested in examining how the quasidistributions approach the light-cone distributions as the meson gets boosted.

All in all, we hope this work can provide some valuable insight on unravelling some gross features of quasidistributions. Moreover, $\mathrm{QCD}_{2}$ may also serve as a benchmark to examine the efficiency among different approaches, e.g., quasidistribution approach versus pseudo-PDF $[37,38]$ and lattice cross section approach [39].

As mentioned earlier, the meson spectra of $\mathrm{QCD}_{2}$ in the large- $N$ limit can be obtained in two equivalent ways.
One is through solving the 't Hooft equation [34], derived from light-cone quantization flavored with the light-cone gauge, while the other is through solving the Bars-Green equations [36], which are inferred from ordinary equal-time quantization combined with the axial gauge. The solutions of the 't Hooft equation correspond to the meson's LCWFs, denoted by $\varphi^{n}(x)$, where $x$ denotes the light-cone momentum fraction carried by the quark relative to that by the meson. $n=0,1, \cdots$ denotes the discrete quantum number, which resembles the principal quantum number $n$ arising from the solution of the Schrödinger equation in onedimensional space. The dimensionless momentum ratio $x$ is restricted in the interval $[0,1]$. In contrast, the solutions of Bars-Green equations are represented by a pair of boundstate wave functions $\varphi_{ \pm}^{n}(k, P)$, where $k=x P$ is the spatial component of momentum carried by the quark and $P$ denotes the meson momentum's spatial component. Here the dimensionless ratio $x$ is completely unbounded, $-\infty<x<\infty$.

In [40], we have numerically solved the Bars-Green equations for a variety of quark mass, and with several different meson momenta. We have explicitly verified the Poincaré invariance of the 't Hooft model in the equal-time quantization, in the sense that meson spectra do not depend on the reference frame. We have also numerically confirmed that, in the infinite momentum frame (IMF), i.e., $P \rightarrow \infty$, the Bars-Green wave functions approach asymptotically

$$
\lim _{P \rightarrow \infty} \varphi_{+}^{n}(x P, P)=\varphi^{n}(x), \quad \lim _{P \rightarrow \infty} \varphi_{-}^{n}(x P, P)=0 .
$$

Note the "negative energy" (backward motion in time) component of the wave functions, $\varphi_{-}$fades away as $P \rightarrow \infty$, while the "positive energy" (forward motion in time) component of the wave functions, $\varphi_{+}$recovers the 't Hooft wave function in the IMF.

Our primary achievement in this work is to construct the light-cone (quasi)distributions out of the 't Hooft wave function (Bars-Green) wave functions, thus developing a concrete feel about the nature of the quasidistributions. Based on the numerical solutions of the wave functions reported in [40], we then quantitatively compare the quasiparton distributions and their light-cone counterparts accordingly. Rather than Wick-rotate into Euclidean spacetime, we stay in the Minkowski spacetime to compute the quasidistributions.

Apart from looking into nonperturbative aspects, we also study the quasidistributions in $\mathrm{QCD}_{2}$ from the angle of perturbation theory. By replacing a meson with a quark (or quark-antiquark pair), we compute these fictitious "mesonic" light-cone PDFs and PDFs (LCDA and quasiDA) to one-loop order and explicitly verify that both of them share the identical IR behavior at the leading order in $1 / P_{z}$. Hence, one of the backbones of LaMET is explicitly validated in this novel theoretical setting. 
The rest of the paper is structured as follows. In Sec. II, we set up the theory of $\mathrm{QCD}_{2}$ and introduce our notations. In Sec. III, we recapitulate the Hamiltonian operator approach to derive the 't Hooft equation, in the context of light-cone quantization flavored with light-cone gauge. Though being an old subject, we feel that there still exists some notable innovation in our derivation. In Sec. IV, in the framework of equal-time quantization flavored with axial gauge, we revisit the derivation of the Bars-Green equations by employing Hamiltonian operator approach, as well as Bogoliubov transformation. The novelty of our derivation is that we adopt momentum cutoff rather than principle value prescription as an alternative means to regularize IR singularities encountered in the intermediate stage. In Sec. V, with the aid of the bosonization procedure, we present an analytic expressions for the light-cone and the quasi-PDFs in terms of the bound-state wave functions. We stress that quasidistributions also depend on an essential quantity, the Bogoliubov-Chiral angle. In Sec. VI, following the same bosonization program, we proceed to present the analytical expressions for the LCDA and quasi-DA. In Sec. VII, we proceed to conduct a comprehensive numerical study for light-cone and quasi-PDFs, DAs related to a variety of meson species: chiral $\pi$, physical pion, a fictitious "strangeonium," and charmonium. For the quasidistributions, we choose several different reference frames for each meson species, to illuminate how their profiles evolve with the increasing meson momentum. In Sec. VIII, we conduct the one-loop perturbative calculation for both light-cone and quasi-PDFs, as well as LCDA and quasi-DA, associated with a fictitious meson, using both covariant and time-ordered perturbation theory. Again with the IR singularities regularized by a soft momentum cutoff, we explicitly examine the IR cutoff dependence of the lightcone and quasidistributions. Finally, we summarize in Sec. IX. In Appendix A, we conduct a comparative study between one variant of the quasi-PDF (DA) and the canonical quasi-PDF (DA), examining which version of quasidistributions evolve to their light-cone counterpart at a faster pace under Lorentz boost. In Appendix B, we present some distribution identities that are useful to express the perturbative LCDA and quasi-DA in terms of the so-called "4-plus" function in Sec. VIII.

\section{SETUP OF THE NOTATIONS}

For simplicity, throughout this work, we will only consider a single flavor of quark. Consequently, we will be interested only in flavor-singlet mesons (quarkonia). Adding more flavors does not pose any principal difficulty, and we will avoid this unnecessary complication. Bearing a local color $S U(N)$ symmetry, the Lagrangian density of the $\mathrm{QCD}_{2}$ reads

$$
\mathcal{L}_{\mathrm{QCD}_{2}}=-\frac{1}{4}\left(F_{\mu \nu}^{a}\right)^{2}+\bar{\psi}(i D-m) \psi,
$$

where $m$ denotes the quark mass. $\psi$ represents the quark field, which contains two components in the Dirac spinor space and $N$ components in the color space. $F_{\mu \nu}^{a} \equiv$ $\partial_{\mu} A_{\nu}^{a}-\partial_{\nu} A_{\mu}^{a}+g_{s} f^{a b c} A_{\mu}^{b} A_{\nu}^{c}$ is the gluon field strength tensor, with $A_{\mu}^{a}$ denoting the gluon field. $D_{\mu}=\partial_{\mu}-i g_{s} A_{\mu}^{a} T^{a}$ denotes the color covariant derivative. Here, $T^{a}$ represent the generators in the fundamental representation of the color $S U(N)$ group, which are $N \times N$ Hermitian matrices satisfying

$$
\begin{aligned}
\operatorname{tr}\left(T^{a} T^{b}\right) & =\frac{\delta^{a b}}{2} \\
\sum_{a} T_{i j}^{a} T_{k l}^{a} & =\frac{1}{2}\left(\delta_{i l} \delta_{j k}-\frac{1}{N} \delta_{i j} \delta_{k l}\right),
\end{aligned}
$$

where $a, b=1,2, \ldots, N^{2}-1$.

Throughout this work, we define the Lorentz two-vector as $x^{\mu}=\left(x^{0}, x^{z}\right)$, with the superscript 0 indicating the temporal component and $z$ indicating the spatial component. ${ }^{1}$ Moreover, we will adhere to the Weyl-chiral representation for the Dirac $\gamma$-matrices,

$$
\gamma^{0}=\sigma_{1}, \quad \gamma^{z}=-i \sigma_{2}, \quad \gamma_{5} \equiv \gamma^{0} \gamma^{z}=\sigma_{3},
$$

where $\sigma_{i}(i=1,2,3)$ are the familiar Pauli matrices. The advantage of choosing this specific representation is to make the chirality projection simpler, since $\gamma_{5}$ (which coincides with the Lorentz boost generator in two dimensions) becomes diagonal in this basis.

In this paper, we will specify the large- $N$ limit of the $\mathrm{QCD}_{2}$ (the 't Hooft model) as

$$
N \rightarrow \infty, \quad \lambda \equiv \frac{g_{s}^{2} N}{4 \pi} \text { fixed, } \quad m \gg g_{s} \sim \frac{1}{\sqrt{N}} .
$$

where $\lambda$ is dubbed the 't Hooft coupling constant. The last condition in Eq. (5) specifies the so-called weak coupling phase [41]. It is necessary to state this clearly in the outset, since the chiral limit and large- $N$ limit do not generally commute. It is only in the weak coupling regime, i.e., the $m \rightarrow 0$ limit imposed after taking the $N \rightarrow \infty$ limit, that the massless "Goldstone" boson (chiral pion) can arise.

\section{HAMILTONIAN APPROACH IN LIGHT-FRONT QUANTIZATION IN LIGHT-CONE GAUGE}

The bound state equation for $\mathrm{QCD}_{2}$ in the light-cone framework was originally derived by 't Hooft in 1974, based

\footnotetext{
${ }^{1}$ It may appear unwieldy to label the spatial index by " $z$ " in a $1+1$-dimensional field theory. The reason we choose the superscript $z$ instead of 1 or $x$ is to keep conformity with the convention adopted by majority of the literature about quasidistributions in four dimensions.
} 
on the Feynman diagrammatic approach [34]. In the following years, the same equation was also reproduced in the light-cone hamiltonian formalism [42-49]. In this section, we rederive the 't Hooft equation from the angle of light-cone quantization once again. The novelty of our derivation is that we adopt a soft momentum cutoff to regularize severe IR divergence encountered in the intermediate stage, rather than the principal value prescription used in most of the preceding literature. Of course, at the end of the day, we will recover the celebrated 't Hooft equation, which is no longer plagued with infrared singularity.

\section{A. The light-front Hamiltonian}

We adopt the widely used Kogut-Soper convention [50] that the light-cone coordinates are defined through $x^{ \pm}=\left(x^{0} \pm x^{z}\right) / \sqrt{2}$. Consequently, only the off-diagonal components of the metric tensor survive, $g_{+_{-}}=g^{+-}=1$, and $x^{ \pm}=x_{\mp}$.

It is convenient to decompose the quark Dirac field $\psi$ into the right-handed ("good") component $\psi_{R}$ and lefthanded ("bad") component $\psi_{L}$, by acting the chirality projectors $\psi_{R, L}=\frac{1 \pm \gamma_{5}}{2} \psi$. Owing to the diagonal form of $\gamma_{5}$ in the Weyl representation, as specified in (4), one can explicitly decompose

$$
\psi=2^{-\frac{1}{4}}\left(\begin{array}{c}
\psi_{R} \\
\psi_{L}
\end{array}\right)
$$

where $\psi_{R, L}$ represent the single-component Grassmann variables.

To facilitate the light-front quantization, it is convenient to reexpress the $\mathrm{QCD}_{2}$ Lagrangian (2) in terms of light-cone coordinates. Furthermore, the theory gets significantly simplified once imposing the light-cone gauge $A^{+a}=0$ :

$$
\begin{aligned}
\mathcal{L}_{\mathrm{QCD}_{2}}= & \frac{1}{2}\left(\partial_{-} A^{-a}\right)^{2}+i\left(\psi_{R}^{\dagger} D_{+} \psi_{R}+\psi_{L}^{\dagger} \partial_{-} \psi_{L}\right) \\
& -\frac{m}{\sqrt{2}}\left(\psi_{L}^{\dagger} \psi_{R}+\psi_{R}^{\dagger} \psi_{L}\right) .
\end{aligned}
$$

As an exhilarating virtue of the noncovariant gauge, the characteristic complication of QCD, the triple and quartic gluon self-interactions are absent in $\mathrm{QCD}_{2}$.

Regarding $x^{+}$as the light-front time, one observes that only the right-handed quark field constitutes the dynamical degree of freedom. From (7), one then derives the EulerLagrange equation for the left-handed fermion field and the gluon field:

$$
\begin{aligned}
\partial_{-}^{2} A^{-a}(x)-g_{s} \psi_{R}^{\dagger}(x) T^{a} \psi_{R}(x) & =0, \\
i \partial_{-} \psi_{L}(x)-\frac{m}{\sqrt{2}} \psi_{R}(x) & =0 .
\end{aligned}
$$

Hence $\psi_{L}$ and $A^{-a}$ are nonpropagating (constrained) degrees of freedom, rather than the canonical variables. Solutions to the equations of motion (8) are

$$
\begin{aligned}
& \psi_{L}\left(x^{+}, x^{-}\right)=\frac{m}{\sqrt{2} i} \int d y^{-} G_{\rho}^{(1)}\left(x^{-}-y^{-}\right) \psi_{R}\left(x^{+}, y^{-}\right), \\
& A^{-a}\left(x^{+}, x^{-}\right) \\
& \quad=g_{s} \int d y^{-} G_{\rho}^{(2)}\left(x^{-}-y^{-}\right) \psi_{R}^{\dagger}\left(x^{+}, y^{-}\right) T^{a} \psi_{R}\left(x^{+}, y^{-}\right),
\end{aligned}
$$

where $G^{(1)}$ and $G^{(2)}$ correspond to the Green functions associated with the differential operators $\partial_{-}$and $\partial_{-}^{2}$, respectively:

$G_{\rho}^{(1)}\left(x^{-}-y^{-}\right)=i \int_{-\infty}^{+\infty} \frac{d k^{+}}{2 \pi} \Theta\left(\left|k^{+}\right|-\rho\right) \frac{e^{-i k^{+}\left(x^{-}-y^{-}\right)}}{k^{+}}$,

$G_{\rho}^{(2)}\left(x^{-}-y^{-}\right)=-\int_{-\infty}^{+\infty} \frac{d k^{+}}{2 \pi} \Theta\left(\left|k^{+}\right|-\rho\right) \frac{e^{-i k^{+}\left(x^{-}-y^{-}\right)}}{\left(k^{+}\right)^{2}}$.

where $\Theta$ signifies the Heaviside step function, and the sharp momentum cutoff $\rho \rightarrow 0^{+}$is introduced as an IR regulator. We put a subscript $\rho$ in the coordinate-space Green function to stress its implicit dependence on the IR cutoff upon Fourier transform. We note that this peculiar regularization scheme has already been used by 't Hooft [34] and Callan, Coote and Gross [51]. Einhorn even interpreted this IR regulator as a gauge parameter [52]. This IR regulator $\rho$ may linger around the intermediate steps, but must drop away in the physical observables such as meson-meson scattering amplitude [51].

Identifying the light-front Hamiltonian from the Lagrangian (7) through the stress tensor $T^{+-}$, eliminating the occurrences of $\psi_{L}$ and $A^{a \mu}$ in line with (9), we finally end with the desired form of the light-front Hamiltonian ${ }^{2}$ :

\footnotetext{
${ }^{2}$ Note that our light-front Hamiltonian differs from the lightcone Hamiltonian defined in some influential paper [53], which are connected via $H_{\mathrm{LC}} \equiv 2 \mathrm{P}^{+} H_{\mathrm{LF}}$. Our $H_{\mathrm{LF}}$ is framedependent, while $H_{\mathrm{LC}}$ in [53] is not, yet carrying mass dimension two.
} 


$$
\begin{aligned}
H_{\mathrm{LF}} \equiv & P^{-}=\int_{x^{+}=\mathrm{const}} d x^{-}\left\{\frac{m^{2}}{2 i} \psi_{R}^{\dagger}\left(x^{-}\right)\right. \\
& \times \int d y^{-} G_{\rho}^{(1)}\left(x^{-}-y^{-}\right) \psi_{R}\left(y^{-}\right) \\
& -\frac{g_{s}^{2}}{2} \sum_{a} \psi_{R}^{\dagger}\left(x^{-}\right) T^{a} \psi_{R}\left(x^{-}\right) \\
& \left.\times \int d y^{-} G_{\rho}^{(2)}\left(x^{-}-y^{-}\right) \psi_{R}^{\dagger}\left(y^{-}\right) T^{a} \psi_{R}\left(y^{-}\right)\right\} .
\end{aligned}
$$

Note only the canonical variable $\psi_{R}$ survives in the light-front Hamiltonian (11). ${ }^{3}$

The canonical quantization rules in equal light-front time are then

$$
\begin{gathered}
\left\{\psi_{R}^{i}\left(x^{+}, x^{-}\right), \psi_{R}^{j \dagger}\left(y^{+}, y^{-}\right)\right\}_{x^{+}=y^{+}}=\delta^{i j} \delta\left(x^{-}-y^{-}\right), \\
\left\{\psi_{R}^{i}\left(x^{+}, x^{-}\right), \psi_{R}^{j}\left(y^{+}, y^{-}\right)\right\}_{x^{+}=y^{+}} \\
=\left\{\psi_{R}^{i \dagger}\left(x^{+}, x^{-}\right), \psi_{R}^{j \dagger}\left(y^{+}, y^{-}\right)\right\}_{x^{+}=y^{+}}=0 .
\end{gathered}
$$

For clarity, we attach the color indices $i, j=1, \ldots, N$ to the $\psi_{R}$ field explicitly.

\section{B. Bosonization}

To quantize (11), one may expand the $\psi_{R}$ field in terms of the annihilation and creation operators:

$\psi_{R}^{i}\left(x^{-}\right)=\int_{0}^{\infty} \frac{d k^{+}}{2 \pi}\left(b^{i}\left(k^{+}\right) e^{-i k^{+} x^{-}}+d^{i \dagger}\left(k^{+}\right) e^{i k^{+} x^{-}}\right)$,

where $i$ is the color index. The Fock vacuum $|0\rangle$ is defined to satisfy

$$
b^{i}\left(k^{+}\right)|0\rangle=d^{i}\left(k^{+}\right)|0\rangle=0
$$

for any non-negative $k^{+}$.

Following the bosonization procedure [42-49], we define the following four color-singlet compound operators:

\footnotetext{
${ }^{3}$ When concentrating on the color-singlet sectors of Fock space, one is allowed to drop the boundary term in the light-cone Hamiltonian, once the spatial size of the system extends to infinity. One can refer to Hornbostel's thesis for a comprehensive and lucid discussion on this issue [54].
}

$$
\begin{aligned}
M\left(k^{+}, p^{+}\right) & \equiv \frac{1}{\sqrt{N}} \sum_{i} d^{i}\left(k^{+}\right) b^{i}\left(p^{+}\right), \\
M^{\dagger}\left(k^{+}, p^{+}\right) & \equiv \frac{1}{\sqrt{N}} \sum_{i} b^{i \dagger}\left(p^{+}\right) d^{i \dagger}\left(k^{+}\right), \\
B\left(k^{+}, p^{+}\right) & \equiv \frac{1}{\sqrt{N}} \sum_{i} b^{i \dagger}\left(k^{+}\right) b^{i}\left(p^{+}\right), \\
D\left(k^{+}, p^{+}\right) & \equiv \frac{1}{\sqrt{N}} \sum_{i} d^{i \dagger}\left(k^{+}\right) d^{i}\left(p^{+}\right) .
\end{aligned}
$$

The commutation relations among $B, D, M$ and $M^{\dagger}$ are

$$
\begin{aligned}
& {\left[M\left(k_{1}^{+}, p_{1}^{+}\right), M^{\dagger}\left(k_{2}^{+}, p_{2}^{+}\right)\right]} \\
& =(2 \pi)^{2} \delta\left(k_{1}^{+}-k_{2}^{+}\right) \delta\left(p_{1}^{+}-p_{2}^{+}\right)+\mathcal{O}\left(\frac{1}{\sqrt{N}}\right), \\
& {\left[M\left(k_{1}^{+}, p_{1}^{+}\right), B\left(k_{2}^{+}, p_{2}^{+}\right)\right]} \\
& \quad=\left[M^{\dagger}\left(k_{1}^{+}, p_{1}^{+}\right), B\left(k_{2}^{+}, p_{2}^{+}\right)\right]=\mathcal{O}\left(\frac{1}{\sqrt{N}}\right), \\
& {\left[M\left(k_{1}^{+}, p_{1}^{+}\right), D\left(k_{2}^{+}, p_{2}^{+}\right)\right]} \\
& \quad=\left[M^{\dagger}\left(k_{1}^{+}, p_{1}^{+}\right), D\left(k_{2}^{+}, p_{2}^{+}\right)\right]=\mathcal{O}\left(\frac{1}{\sqrt{N}}\right), \\
& {\left[B\left(k_{1}^{+}, p_{1}^{+}\right), B\left(k_{2}^{+}, p_{2}^{+}\right)\right]} \\
& \quad=\left[D\left(k_{1}^{+}, p_{1}^{+}\right), D\left(k_{2}^{+}, p_{2}^{+}\right)\right]=\mathcal{O}\left(\frac{1}{\sqrt{N}}\right), \\
& \quad\left[B\left(k_{1}^{+}, p_{1}^{+}\right), D\left(k_{2}^{+}, p_{2}^{+}\right)\right]=0 .
\end{aligned}
$$

Substituting the Fourier expansion of $\psi_{R}$, (13), into the LF Hamiltonian (11), then expressing everything in terms of the bosonic compound operators introduced in (15), dropping terms that are suppressed by powers of $1 / N$ (with the aid of the $S U(N)$ identity (B3), we can decompose the light-front Hamiltonian into three pieces:

$$
H_{\mathrm{LF}}=H_{\mathrm{LF} ; 0}+: H_{\mathrm{LF} ; 2}:+: H_{\mathrm{LF} ; 4}:
$$

where :: denotes the standard normal ordering. Organized by the frequency of occurrences of the bosonic compound operators, these three parts turn out to be

$$
\begin{aligned}
H_{\mathrm{LF} ; 0}= & N \int \frac{d x^{-}}{2 \pi}\left[\frac{\lambda}{2}+\frac{\lambda-m^{2}}{2} \int_{\rho}^{\infty} \frac{d k^{+}}{k^{+}}\right], \\
: H_{\mathrm{LF} ; 2}:= & \sqrt{N} \int_{\rho}^{\infty} \frac{d k^{+}}{2 \pi}\left[\frac{m^{2}-2 \lambda}{2} \frac{1}{k^{+}}+\frac{\lambda}{\rho}\right] \\
& \times\left[B\left(k^{+}, k^{+}\right)+D\left(k^{+}, k^{+}\right)\right],
\end{aligned}
$$




$$
\begin{aligned}
: H_{\mathrm{LF} ; 4}:= & \frac{\lambda}{8 \pi^{2}} \int_{0}^{\infty} d k_{1}^{+} \int_{0}^{\infty} d k_{2}^{+} \int_{0}^{\infty} d k_{3}^{+} \int_{0}^{\infty} d k_{4}^{+} \\
& \times\left\{\Theta\left(\left|k_{1}^{+}-k_{2}^{+}\right|-\rho\right) \frac{M^{\dagger}\left(k_{2}^{+}, k_{3}^{+}\right) D\left(k_{4}^{+}, k_{1}^{+}\right)-B\left(k_{1}^{+}, k_{4}^{+}\right) M\left(k_{3}^{+}, k_{2}^{+}\right)}{\left(k_{1}^{+}-k_{2}^{+}\right)^{2}} \delta\left(k_{1}^{+}-k_{2}^{+}-k_{3}^{+}-k_{4}^{+}\right)\right. \\
& +\Theta\left(\left|k_{1}^{+}-k_{2}^{+}\right|-\rho\right) \frac{D\left(k_{2}^{+}, k_{3}^{+}\right) M\left(k_{1}^{+}, k_{4}^{+}\right)-M^{\dagger}\left(k_{4}^{+}, k_{1}^{+}\right) B\left(k_{3}^{+}, k_{2}^{+}\right)}{\left(k_{1}^{+}-k_{2}^{+}\right)^{2}} \delta\left(k_{1}^{+}-k_{2}^{+}+k_{3}^{+}+k_{4}^{+}\right) \\
& -\Theta\left(\left|k_{1}^{+}-k_{2}^{+}\right|-\rho\right) \frac{M^{\dagger}\left(k_{1}^{+}, k_{4}^{+}\right) M\left(k_{2}^{+}, k_{3}^{+}\right)+M^{\dagger}\left(k_{2}^{+}, k_{3}^{+}\right) M\left(k_{1}^{+}, k_{4}^{+}\right)}{\left(k_{1}^{+}-k_{2}^{+}\right)^{2}} \delta\left(k_{1}^{+}-k_{2}^{+}-k_{3}^{+}+k_{4}^{+}\right) \\
& +\Theta\left(\left|k_{1}^{+}+k_{2}^{+}\right|-\rho\right) \frac{D\left(k_{4}^{+}, k_{1}^{+}\right) B\left(k_{3}^{+}, k_{2}^{+}\right)+B\left(k_{1}^{+}, k_{4}^{+}\right) D\left(k_{2}^{+}, k_{3}^{+}\right)}{\left(k_{1}^{+}+k_{2}^{+}\right)^{2}} \delta\left(k_{1}^{+}+k_{2}^{+}-k_{3}^{+}-k_{4}^{+}\right) \\
& +\Theta\left(\left|k_{1}^{+}+k_{2}^{+}\right|-\rho\right) \frac{M^{\dagger}\left(k_{4}^{+}, k_{1}^{+}\right) D\left(k_{2}^{+}, k_{3}^{+}\right)-B\left(k_{3}^{+}, k_{2}^{+}\right) M\left(k_{1}^{+}, k_{4}^{+}\right)}{\left(k_{1}^{+}+k_{2}^{+}\right)^{2}} \delta\left(k_{1}^{+}+k_{2}^{+}-k_{3}^{+}+k_{4}^{+}\right) \\
& +\Theta\left(\left|k_{1}^{+}+k_{2}^{+}\right|-\rho\right) \frac{D\left(k_{4}^{+}, k_{1}^{+}\right) M\left(k_{3}^{+}, k_{2}^{+}\right)-M^{\dagger}\left(k_{2}^{+}, k_{3}^{+}\right) B\left(k_{1}^{+}, k_{4}^{+}\right)}{\left(k_{1}^{+}+k_{2}^{+}\right)^{2}} \delta\left(k_{1}^{+}+k_{2}^{+}+k_{3}^{+}-k_{4}^{+}\right) \\
& \left.-\Theta\left(\left|k_{1}^{+}-k_{2}^{+}\right|-\rho\right) \frac{D\left(k_{2}^{+}, k_{3}^{+}\right) D\left(k_{4}^{+}, k_{1}^{+}\right)+B\left(k_{3}^{+}, k_{2}^{+}\right) B\left(k_{1}^{+}, k_{4}^{+}\right)}{\left(k_{1}^{+}-k_{2}^{+}\right)^{2}} \delta\left(k_{1}^{+}-k_{2}^{+}+k_{3}^{+}-k_{4}^{+}\right)\right\},
\end{aligned}
$$

where $\lambda=g_{s}^{2} N / 4 \pi$ is the dimensional 't Hooft coupling constant, and those terms suppressed by $1 / N$ have been suppressed. $H_{\mathrm{LF} ; 0}$ can be interpreted as the vacuum light-cone energy, which is both UV and IR divergent [55]. This constant is irrelevant for our purpose, so will be neglected henceforth. Note the soft momentum cutoff $\rho$ has been introduced in (18) to regularize the IR divergence.

A key observation is that the $\mathrm{QCD}_{2}$ is a confining theory, and one cannot create or annihilate isolated quarks and antiquarks. Therefore, to create a quark, one has to create an accompanying antiquark; vice versa, to annihilate a quark, one has to annihilate an accompanying antiquark. Only the color-singlet $q \bar{q}$ pair can be created or annihilated. The consequence is that the operators in (15) cannot be all independent. Rather one finds that the compound operators $B$ and $D$ can be built out of $M$ and $M^{\dagger}$ [49]:

$$
\begin{aligned}
& B\left(k^{+}, p^{+}\right) \rightarrow \frac{1}{\sqrt{N}} \int_{0}^{\infty} \frac{d q^{+}}{2 \pi} M^{\dagger}\left(q^{+}, k^{+}\right) M\left(q^{+}, p^{+}\right), \\
& D\left(k^{+}, p^{+}\right) \rightarrow \frac{1}{\sqrt{N}} \int_{0}^{\infty} \frac{d q^{+}}{2 \pi} M^{\dagger}\left(k^{+}, q^{+}\right) M\left(p^{+}, q^{+}\right) .
\end{aligned}
$$

As can be readily verified, these relations are compatible with the commutation relation (16).

Substituting (19) into (18), relabelling the momenta $p^{+}=x P^{+}$and $k^{+}=(1-x) P^{+}$, keeping only the leading order terms in $1 / N$, one finds that the $: H_{\mathrm{LF} ; 2}:$ and $: H_{\mathrm{LF} ; 4}:$ components now read

$$
\begin{aligned}
: H_{\mathrm{LF} ; 2}:= & \frac{1}{(2 \pi)^{2}} \int_{\rho}^{\infty} d P^{+} \int_{0}^{1} d x M^{\dagger}\left((1-x) P^{+}, x P^{+}\right) M\left((1-x) P^{+}, x P^{+}\right) \\
& \times\left\{\left[\left(\frac{m^{2}}{2}-\lambda\right) \frac{1}{x}+\frac{P^{+} \lambda}{\rho}\right] \Theta\left(x-\frac{\rho}{P^{+}}\right)+\left[\left(\frac{m^{2}}{2}-\lambda\right) \frac{1}{1-x}+\frac{P^{+} \lambda}{\rho}\right] \Theta\left(1-\frac{\rho}{P^{+}}-x\right)\right\}, \\
: H_{\mathrm{LF} ; 4}:= & -\frac{\lambda}{(2 \pi)^{2}} \int_{\rho}^{\infty} d P^{+} \int_{0}^{1} \int_{0}^{1} d x d y \Theta\left(|x-y|-\frac{\rho}{P^{+}}\right) \frac{1}{(x-y)^{2}} \times M^{\dagger}\left((1-x) P^{+}, x P^{+}\right) M\left((1-y) P^{+}, y P^{+}\right) .
\end{aligned}
$$

\section{Diagonalization, principal value prescription, and the 't Hooft equation}

Our goal is to diagonalize the light-front hamiltonian (20). To this purpose, it is convenient to introduce an infinite set of meson annihilnation/creation operators: $m_{n}\left(P^{+}\right) / m_{n}^{\dagger}\left(P^{+}\right)$, where $n$ stands for the principal quantum number, and $P^{+}$represents the light-cone momentum of the corresponding meson. We postulate that the $M$ operator basis is connected to the $m_{n}$ basis through 


$$
\begin{aligned}
& M\left((1-x) P^{+}, x P^{+}\right)=\sqrt{\frac{2 \pi}{P^{+}}} \sum_{n=0}^{\infty} \varphi_{n}(x) m_{n}\left(P^{+}\right), \\
& m_{n}\left(P^{+}\right)=\sqrt{\frac{P^{+}}{2 \pi}} \int_{0}^{1} d x \varphi_{n}(x) M\left((1-x) P^{+}, x P^{+}\right),
\end{aligned}
$$

where $\varphi_{n}(x)$ is understood to be the $n$th coefficient function. The physical picture is clear, since confinement nature of 't Hooft model, exciting a quarkantiquark pair from the Fock vacuum would eventually lead to the formation of a meson, in the large- $N$ limit. To the best of our knowledge, the explicit writing of the decomposition formula (21) is new.

We postulate that the mesonic annihilation and creation operators $m_{n}$ and $m_{n}^{\dagger}$ obey the canonical commutation relations:

$$
\left[m_{n}\left(P_{1}^{+}\right), m_{r}^{\dagger}\left(P_{2}^{+}\right)\right]=2 \pi \delta_{n r} \delta\left(P_{1}^{+}-P_{2}^{+}\right)
$$

and all other commutators vanish. It is straightforward to check that, in order to satisfy these commutation relations, the coefficient functions $\varphi_{n}(x)$ must be subject to the following orthogonality and completeness conditions:

$$
\begin{aligned}
\int_{0}^{1} d x \varphi_{n}(x) \varphi_{m}(x) & =\delta_{n m}, \\
\sum_{n} \varphi_{n}(x) \varphi_{n}(y) & =\delta(x-y),
\end{aligned}
$$

We wish that the light-front Hamiltonian in the basis of $m_{n}$ and $m_{n}^{\dagger}$ operators is in a diagonal form,

$$
H_{\mathrm{LF}}=H_{\mathrm{LF} ; 0}+\int \frac{d P^{+}}{2 \pi} P_{n}^{-} m_{n}^{\dagger}\left(P^{+}\right) m_{n}\left(P^{+}\right)
$$

where $P^{-}$is the light-cone energy of the $n$th mesonic state, $P_{n}^{-}=M_{n}^{2} /\left(2 P^{+}\right)$.

If the light-front Hamiltonian can be diagonalized in the new $m_{n}$ operator basis, the $n$th mesonic state in the large- $N$ limit can be directly constructed via

$$
\left|P_{n}^{-}, P^{+}\right\rangle=\sqrt{2 P^{+}} m_{n}^{\dagger}\left(P^{+}\right)|0\rangle .
$$

In order to reach the desired form (24), one should enforce the condition that the coefficients of all the unwanted operators of the form $m_{n}^{\dagger} m_{r}(n \neq r)$ vanish. This requirement leads to the following equations which must be satisfied by $\varphi_{n}(x)$ in different intervals of $x$ :

$$
\begin{aligned}
& \frac{m^{2}}{1-x} \varphi_{n}(x)-2 \lambda \int_{x+\frac{\rho}{P^{+}}}^{1} d y \frac{\varphi_{n}(y)-\varphi_{n}(x)}{(x-y)^{2}} \\
& =M_{n}^{2} \varphi_{n}(x) \quad 0<x<\frac{\rho}{P^{+}}, \\
& \left(\frac{m^{2}}{x}+\frac{m^{2}}{1-x}\right) \varphi_{n}(x)-2 \lambda \int_{0}^{1} d y \Theta(|x-y|-\rho) \frac{\varphi_{n}(y)-\varphi_{n}(x)}{(x-y)^{2}} \\
& =M_{n}^{2} \varphi_{n}(x) \quad \frac{\rho}{P^{+}}<x<1-\frac{\rho}{P^{+}}, \\
& \frac{m^{2}}{x} \varphi_{n}(x)-2 \lambda \int_{0}^{x-\frac{\rho}{P^{+}}} d y \frac{\varphi_{n}(y)-\varphi_{n}(x)}{(x-y)^{2}} \\
& =M_{n}^{2} \varphi_{n}(x) \quad 1-\frac{\rho}{P^{+}}<x<1,
\end{aligned}
$$

In the $\rho \rightarrow 0^{+}$limit, these equations merge into a single equation:

$$
\begin{aligned}
& \left(\frac{m^{2}}{x}+\frac{m^{2}}{1-x}\right) \varphi_{n}(x)-2 \lambda f_{0}^{1} d y \frac{\varphi_{n}(y)-\varphi_{n}(x)}{(x-y)^{2}} \\
& =M_{n}^{2} \varphi_{n}(x), \quad 0<x<1,
\end{aligned}
$$

where the dashed integral $f$ in (27) denotes the principal value $(\mathrm{PV})$ prescription:

$f d y \frac{f(y)}{(x-y)^{2}}=\lim _{\epsilon \rightarrow 0^{+}} \int d y \Theta(|x-y|-\epsilon) \frac{f(y)-f(x)}{(x-y)^{2}}$.

with $f(y)$ a test function that is regular at $y=x$.

Eq. (27) is nothing but the celebrated 't Hooft equation. Now the coefficient functions $\varphi_{n}(x)$, first introduced in (21), can be interpreted as the 't Hooft wave function, or the light-cone wave function of the $n$th mesonic state.

We emphasize that the PV prescription as specified in (27) needs not be unique. Here we just list two additional popular PV prescriptions:

$$
\begin{aligned}
& f d y \frac{f(y)}{(x-y)^{2}} \\
& \quad=\lim _{\epsilon \rightarrow 0} \int d y \frac{f(y)}{2}\left[\frac{1}{(x-y+i \epsilon)^{2}}+\frac{1}{(x-y-i \epsilon)^{2}}\right], \\
& f_{d y} \frac{f(y)}{(x-y)^{2}}=\lim _{\epsilon \rightarrow 0^{+}} \int d y \Theta(|x-y|-\epsilon) \frac{f(y)}{(x-y)^{2}}-\frac{2 f(x)}{\epsilon},
\end{aligned}
$$

where the first one was adopted in [52] (also referred to as Mandelstam-Leibbrandt prescription [56,57]), and the second one was introduced by Hadamard long ago [58]. All the aforementioned PV prescriptions are mathematically equivalent, but may practically differ in efficiency upon numerical implementation. 


\section{HAMILTONIAN APPROACH IN EQUAL-TIME QUANTIZATION IN AXIAL GAUGE}

The bound-state equations in $\mathrm{QCD}_{2}$ in equal-time quantization and in axial gauge were originally derived by Bars and Green in 1978 [36], largely utilizing Feynman diagrammatic techniques. In 2001, Kalashnikova and Nefediev presented an elegant derivation based on the Hamiltonian operator approach [59]. A nice feature of this method is that, through introducing Bogoliubov transformation, the physical meaning of the Bars-Green wave functions is greatly clarified.

It appears rather obscure to link the Bars-Green boundstate wave functions with the quasidistributions based on the diagrammatic methods. On the contrary, it is quite transparent to achieve this goal with the aid of the bosonization technique. Therefore, it is rewarding to recapitulate the derivation of the Bars-Green equations in this section, again within the Hamiltonian approach.

Reference [59] employs the PV prescription to sweep away the potential IR divergences. Nevertheless, to be compatible with our treatment in light-front quantization in Sec. III as well as in the perturbative one-loop computation for quasidistributions in Sec. VIII, here we adopt the same momentum cutoff as the IR regulator. We will explicitly verify that, though differing in intermediate steps, after the IR momentum cutoff is removed in the end, the famous mass gap equation and Bars-Green equations will be recovered.

\section{A. The Hamiltonian in the axial gauge}

Enforcing the axial gauge condition $A^{z}=0$, the $\mathrm{QCD}_{2}$ Lagrangian in (2) reduces to

$$
\mathcal{L}_{\mathrm{QCD}_{2}}=\frac{1}{2}\left(\partial_{z} A_{0}^{a}\right)^{2}+i \psi^{\dagger}\left(D_{0}+\gamma^{5} \partial_{z}\right) \psi-m \bar{\psi} \psi .
$$

Unlike the light-cone case, both components of the quark Dirac field $\psi$ remain as the propagating degrees of freedom. The equation of motion for $A^{0 a}$ 's turns out to be

$$
\partial_{z}^{2} A^{0 a}=g_{s} \psi^{\dagger} T^{a} \psi
$$

thus, $A^{0 a}$ is a constrained rather than dynamical variable.

The solution of Eq. (31) is

$$
A^{0 a}(t, z)=g_{s} \int d z^{\prime} \tilde{G}_{\rho}^{(2)}\left(z-z^{\prime}\right) \psi^{\dagger}\left(t, z^{\prime}\right) T^{a} \psi\left(t, z^{\prime}\right),
$$

where $\tilde{G}_{\rho}^{(2)}$ denotes the Green function associated with the operator $\partial_{z}^{24}$ :

\footnotetext{
${ }^{4}$ For notational brevity, in this section, we have suppressed the superscript " $z$ " for the spatial component of a 2 -vector, so $k$ should be understood as $k^{z}$ if no confusion arises.
}

$$
\tilde{G}_{\rho}^{(2)}\left(z-z^{\prime}\right)=-\int_{-\infty}^{+\infty} \frac{d k}{2 \pi} \Theta(|k|-\rho) \frac{e^{i k\left(z-z^{\prime}\right)}}{k^{2}} .
$$

This Fourier integral is ill defined due to the singularity caused by $k \rightarrow 0$. For consistency with the rest of the paper, we again employ a momentum cutoff $\rho \rightarrow 0^{+}$to regularize the IR divergence.

The equal-time Hamiltonian in the axial gauge, when expressed in terms of the canonical variables, is ${ }^{5}$

$$
\begin{aligned}
H \equiv & P^{0}=\int_{t=\mathrm{const}} d z\left\{\psi^{\dagger}(z)\left(-i \gamma^{5} \partial_{z}+m \gamma^{0}\right) \psi(z)\right. \\
& \left.-\frac{g_{s}^{2}}{2} \sum_{a} \int d z^{\prime} \psi^{\dagger}(z) T^{a} \psi(z) \tilde{G}_{\rho}^{(2)}\left(z-z^{\prime}\right) \psi^{\dagger}\left(z^{\prime}\right) T^{a} \psi\left(z^{\prime}\right)\right\} .
\end{aligned}
$$

The equal-time canonical quantization rule is then

$$
\begin{aligned}
& \left\{\psi^{i}(t, z), \psi^{j \dagger}\left(t^{\prime}, z^{\prime}\right)\right\}_{t=t^{\prime}}=\delta_{i j} \delta\left(z-z^{\prime}\right), \\
& \left\{\psi^{i}(t, z), \psi^{j}\left(t^{\prime}, z^{\prime}\right)\right\}_{t=t^{\prime}}=\left\{\psi^{i \dagger}(z), \psi^{j \dagger}\left(z^{\prime}\right)\right\}_{t=t^{\prime}}=0,
\end{aligned}
$$

where $i, j=1, \ldots, N$ denote the color indices carried by $\psi$, and the spinor indices have been suppressed for simplicity.

\section{B. Dressed quark basis and mass-gap equation}

To proceed, we expand the Dirac $\psi$ field in terms of the quark annihilation and creation operators,

$\psi^{i}(z)=\int \frac{d p}{2 \pi} \frac{1}{\sqrt{2 \tilde{E}(p)}}\left[b^{i}(p) u(p)+d^{i \dagger}(-p) v(-p)\right] e^{i p z}$,

where the factor $\sqrt{2 \tilde{E}(p)}$ is deliberately inserted in the integration measure, to keep our normalization convention compatible with the standard text [60]. Here, $\tilde{E}(p)$ can be interpreted as the energy carried by the dressed quark. The spinor wave functions $u, v$ are parametrized as ${ }^{6}$

$u(p)=\sqrt{\tilde{E}(p)} T(p)\left(\begin{array}{l}1 \\ 1\end{array}\right), \quad v(-p)=\sqrt{\tilde{E}(p)} T(p)\left(\begin{array}{c}1 \\ -1\end{array}\right)$,

where $T(p)$ is a unitary $2 \times 2$ matrix. In conformity with the convention adopted in [60], the $u, v$ spinor wave functions carry the mass dimension of $\frac{1}{2}$. Combining these two

\footnotetext{
${ }^{5}$ Bars and Green expounded why one is allowed to drop the boundary terms in the color-singlet sector, in the context of equaltime quantization and axial gauge [36].

${ }^{6}$ Note here the parametrization of the dressed quark spinor wave functions differs from the preceding literature [36,59], where the Dirac-Pauli representation for $\gamma$-matrices were adopted.
} 
equations, one sees that the field expansion in (36) actually does not rely on the explicit form of $\tilde{E}(p)$ at all, but critically depends on the dressing function $T(p)$.

The quark vacuum state is defined to be

$$
b^{i}(k)|0\rangle=d^{i}(k)|0\rangle=0
$$

for all possible values of $k$.

Substituting the Fourier expansion of $\psi$ (36), into the Hamiltonian in (34), and rearranging it into the normalordered form, we can decompose the Hamiltonian into three pieces:

$$
H=H_{0}+: H_{2}:+: H_{4}:
$$

which contain 0,2 , and 4 quark creation/annihilation operators accordingly,

$$
\begin{aligned}
H_{0}= & N \int d z \int \frac{d p}{2 \pi} \operatorname{Tr}\left[\left(p \gamma^{5}+m \gamma^{0}\right) \Lambda_{-}(p)\right. \\
+ & \left.\frac{\lambda}{2} \int \frac{d k}{(k-p)^{2}} \Theta(|k-p|-\rho) \Lambda_{+}(p) \Lambda_{-}(k)\right], \\
: H_{2}:= & \int d p \operatorname{Tr}\left[\Xi(p) \Lambda_{+}(p) b^{i \dagger}(p) b^{i}(p)\right. \\
& +\Xi(p) \Omega_{-}(p) b^{i \dagger}(p) d^{i \dagger}(-p) \\
& +\Xi(p) \Omega_{+}(p) d^{i}(-p) b^{i}(p) \\
& \left.-\Xi(p) \Lambda_{-}(p) d^{i \dagger}(-p) d^{i}(-p)\right] \\
: H_{4}:= & -\frac{g_{s}^{2}}{2} \sum_{a} \iint_{t=\text { const }} d z d z^{\prime} \\
: \psi^{\dagger}(z) & T^{a} \psi(z) \tilde{G}_{\rho}^{(2)}\left(z-z^{\prime}\right) \psi^{\dagger}\left(z^{\prime}\right) T^{a} \psi\left(z^{\prime}\right):
\end{aligned}
$$

where the matrices $\Xi, \Lambda_{ \pm}$, and $\Omega_{ \pm}$are defined as

$$
\begin{gathered}
\Xi(p)=p \gamma^{5}+m \gamma^{0} \\
+\sum_{i} \frac{\lambda}{2} \int \frac{d k}{2 \pi(p-k)^{2}} \Theta(|p-k|-\rho)\left(\Lambda_{+}(k)-\Lambda_{-}(k)\right), \\
\Lambda_{ \pm}(k)=T(k) \frac{1 \pm \gamma^{0}}{2} T^{\dagger}(k), \\
\Omega_{ \pm}(k)=T(k) \frac{\gamma^{0} \pm 1}{2} \gamma^{z} T^{\dagger}(k) .
\end{gathered}
$$

$H_{0}$ describes the vacuum energy. Let us first focus on the single dressed quark sector represented by $: \mathrm{H}_{2}:$. We are seeking a possible solution of $T(p)$ such that $: \mathrm{H}_{2}$ : has a diagonalized form in the basis of quark annihilation and creation operators,
$: H_{2}:=\sum_{i} \int_{-\infty}^{\infty} \frac{d k}{2 \pi} \tilde{E}_{i}(k)\left(b^{i \dagger}(k) b^{i}(k)+d^{i \dagger}(k) d^{i}(k)\right)$,

where $i$ is the color index, and $\tilde{E}_{i}(k)$ denotes the energy of the dressed quark with momentum $k$. To proceed, one parametrizes the $T(p)$ as [36]

$$
T(p)=\exp \left[-\frac{1}{2} \theta(p) \gamma^{z}\right]
$$

where $\theta(p)$ is called the Bogliubov-chiral angle, which is an odd function of $p$ [36,59]. As elucidated in Ref. [61], $T(p)$ is reminiscent of the Foldy-Wouthuysen transformation that decouples the positive and negative energy degrees of freedom in Dirac field, and $\theta(p)$ play the role of the Foldy-Wouthuysen angle.

By the parametrization specified in (43), diagonalization of (40b) leads to two coupled equations for $\theta(p)$ and $\tilde{E}(p)$, respectively,

$\tilde{E}(p) \cos \theta(p)=m+\frac{\lambda}{2} \int \frac{d k}{(k-p)^{2}} \Theta(|k-p|-\rho) \cos \theta(k)$,

$\tilde{E}(p) \sin \theta(p)=p+\frac{\lambda}{2} \int \frac{d k}{(k-p)^{2}} \Theta(|k-p|-\rho) \sin \theta(k)$.

After some plain linear algebra on two equations in (44), we finally arrive at the nonlinear equation for $\theta(p)$,

$$
\begin{aligned}
& p \cos \theta(p)-m \sin \theta(p) \\
& =\frac{\lambda}{2} \lim _{\rho \rightarrow 0^{+}} \int_{-\infty}^{+\infty} \frac{d k}{(p-k)^{2}} \Theta(|k-p|-\rho) \sin [\theta(p)-\theta(k)],
\end{aligned}
$$

which is nothing but the celebrated mass-gap equation [36]. Note that the limit $\rho \rightarrow 0^{+}$just serves the standard Cauchy principal value prescription. Examining the gap equation (45), the interpretation of $\theta(p)$ as the FoldyWouthuysen angle becomes transparent if the interaction term, which is directly responsible for dressing the bare quark, can be temporarily turned off. The angle $\theta(p)$ plays a vital role for generating a nonvanishing quark vacuum condensate. Practically speaking, the Bogoliubov-chiral angle can only be solved numerically, even in the chiral limit.

In passing, we stress that the mass-gap equation (45) can be obtained from another quite different perspective. Rather than diagonalize $: H_{2}:$, one can take a closer look at the vacuum energy constant. One can rewrite (40a) as 


$$
\begin{aligned}
\mathcal{E}_{\mathrm{vac}}[\theta(p)]= & N \int \frac{d p}{2 \pi}\{-m \cos \theta(p)-p \sin \theta(p) \\
& \left.+\frac{\lambda}{2} \int d k \Theta(|k-p|-\rho) \frac{1-\cos [\theta(k)-\theta(p)]}{2(k-p)^{2}}\right\},
\end{aligned}
$$

where $\mathcal{E}_{\mathrm{vac}}=H_{0} / L$ is the vacuum energy density, with $L$ the length of the spatial interval.

Minimizing Eq. (46) with respect to $\theta(p)$, one can readily obtain a variational equation, which exactly reproduce Eq. (45) [59]. Note that the true vacuum is no longer chiral invariant, and a nonzero quark condensate arises in the chiral limit, which signals the spontaneous chiral symmetry breaking in large- $N$ limit of $\mathrm{QCD}_{2}$ [62].

Once the Bogoliubov angle $\theta(p)$ is known, one can then infer the dispersive law for a dressed quark:

$$
\begin{aligned}
\tilde{E}(p)= & m \cos \theta(p)+p \sin \theta(p) \\
& +\frac{\lambda}{2} \int_{-\infty}^{+\infty} \frac{d k}{(p-k)^{2}} \Theta(|k-p|-\rho) \cos [\theta(p)-\theta(k)] .
\end{aligned}
$$

It is straightforward to see that the energy carried by the dressed quark blows up for all values of momentum, $\tilde{E}(p) \rightarrow \frac{\lambda}{\rho}$, after the IR regulator is removed. This symptom is in sharp contrast to the regular dispersive law obtained in [36,62], where the PV scheme is used to regularize the IR divergence there. As a consequence, the free Hamiltonian in the dressed quark sector in (42) is ill defined, due to its sensitivity to the IR cutoff. Nevertheless, this is a harmless and tolerable nuisance, since the colored object such as dressed quark need not be affiliated with any physical significance.

For future usage, it is convenient to define the regularized dressed quark energy, $E(p)$ :

$$
\begin{aligned}
E(p) \equiv & \tilde{E}(p)-\frac{\lambda}{\rho}=m \cos \theta(p)+p \sin \theta(p) \\
& +\frac{\lambda}{2} f_{-\infty}^{+\infty} \frac{d k}{(p-k)^{2}} \cos [\theta(p)-\theta(k)],
\end{aligned}
$$

where $f$ denotes the PV scheme as specified in (28). It is straightforward to see that $E(p)$ is an even function of $p$, and remain finite for all finite $p$. Nevertheless, being a colored object, the dispersive relation for a dressed quark, no matter $\widetilde{E}(p)$ or $E(p)$, clearly violates Lorentz covariance.

\section{Bosonization}

In order to derive the bound state equation, we must take the interaction part of the Hamiltonian, $: H_{4}:$, into account. In parallel with the bosonization procedure for the LF
Hamiltonian, here we introduce the following color-singlet compound operators analogous to (15):

$M(p, q) \equiv \frac{1}{\sqrt{N}} \sum_{i} d_{-p}^{i} b_{q}^{i}, \quad M^{\dagger}(p, q) \equiv \frac{1}{\sqrt{N}} \sum_{i} b_{q}^{i \dagger} d_{-p}^{i \dagger}$,

$B(p, q) \equiv \frac{1}{\sqrt{N}} \sum_{i} b_{p}^{i \dagger} b_{q}^{i}, \quad D(p, q) \equiv \frac{1}{\sqrt{N}} \sum_{i} d_{-p}^{i \dagger} d_{-q}^{i}$.

The commutation relations among $M, M^{\dagger}, B$, and $D$ in the large- $N$ limit are

$$
\begin{gathered}
{\left[M\left(k_{1}, p_{1}\right), M^{\dagger}\left(k_{2}, p_{2}\right)\right]} \\
=(2 \pi)^{2} \delta\left(k_{1}-k_{2}\right) \delta\left(p_{1}-p_{2}\right)+\mathcal{O}\left(\frac{1}{\sqrt{N}}\right), \\
{\left[M\left(k_{1}, p_{1}\right), B\left(k_{2}, p_{2}\right)\right]} \\
=\left[M^{\dagger}\left(k_{1}, p_{1}\right), B\left(k_{2}, p_{2}\right)\right]=\mathcal{O}\left(\frac{1}{\sqrt{N}}\right), \\
{\left[M\left(k_{1}^{+}, p_{1}^{+}\right), D\left(k_{2}, p_{2}\right)\right]} \\
=\left[M^{\dagger}\left(k_{1}, p_{1}\right), D\left(k_{2}, p_{2}\right)\right]=\mathcal{O}\left(\frac{1}{\sqrt{N}}\right), \\
{\left[B\left(k_{1}, p_{1}\right), B\left(k_{2}, p_{2}\right)\right]} \\
=\left[D\left(k_{1}, p_{1}\right), D\left(k_{2}, p_{2}\right)\right]=\mathcal{O}\left(\frac{1}{\sqrt{N}}\right), \\
\quad\left[B\left(k_{1}, p_{1}\right), D\left(k_{2}, p_{2}\right)\right]=0,
\end{gathered}
$$

which are very similar to their light-cone counterparts (16).

Due to the confinement nature of $\mathrm{QCD}_{2}$, the same consideration that leads to (19) can also be applied here, i.e., not all compound operators in (49) are independent. In fact, one finds that [59]

$$
\begin{aligned}
& B\left(p, p^{\prime}\right)=\frac{1}{\sqrt{N}} \int_{-\infty}^{+\infty} \frac{d q}{2 \pi} M^{\dagger}(q, p) M\left(q, p^{\prime}\right), \\
& D\left(p, p^{\prime}\right)=\frac{1}{\sqrt{N}} \int_{-\infty}^{+\infty} \frac{d q}{2 \pi} M^{\dagger}(p, q) M\left(p^{\prime}, q\right) .
\end{aligned}
$$

Here we follow similar steps as what lead to (18) in lightcone quantization. Substituting the Fourier expansion of $\psi$, (36), into the Hamiltonian (40), then expressing everything in terms of the bosonic compound operators introduced in (49), eliminating $B, D$ in line with (51), and only keeping terms at leading order in $1 / \mathrm{N}$, the $: H_{2}:$ and $: H_{4}:$ pieces in (39) read 


$$
\begin{aligned}
: H_{2}:= & \iint \frac{d P d p}{(2 \pi)^{2}}(\tilde{E}(p)+\tilde{E}(P-p)) \\
& \times M^{\dagger}(p-P, p) M(p-P, p), \\
: H_{4}:= & -\frac{\lambda}{8 \pi^{2}} \int d P \iint \frac{d p d k}{(p-k)^{2}} \Theta(|p-k|-\rho) \\
& \times\left\{2 C(p, k, P) M^{\dagger}(p-P, p) M(k-P, k),\right. \\
& +S(p, k, P)[M(p, p-P) M(k-P, k) \\
& \left.\left.+M^{\dagger}(p, p-P) M^{\dagger}(k-P, k)\right]\right\},
\end{aligned}
$$

where the functions $S$ and $C$ are defined as [36]

$$
\begin{aligned}
& C(p, k, P)=\cos \frac{\theta(p)-\theta(k)}{2} \cos \frac{\theta(P-p)-\theta(P-k)}{2}, \\
& S(p, k, P)=\sin \frac{\theta(p)-\theta(k)}{2} \sin \frac{\theta(P-p)-\theta(P-k)}{2} .
\end{aligned}
$$

\section{Bogoliubov transformation, diagonalization, and Bars-Green equations}

The Hamiltonian $: H_{2}:+: H_{4}:$ in (52) is not yet in the diagonalized form. Parametrically, it bears the specific structure,

$$
H \sim H_{0}+A M^{\dagger} M+B\left(M^{\dagger} M^{\dagger}+M M\right),
$$

which is reminiscent of the Hamiltonian for the dilute weakly interacting Bose gas [63]. The familiar strategy of diagonalizing this type of Hamiltonian is through the Bogoliubov transformation [63]:

$$
\begin{gathered}
m=u M+v M^{\dagger}, \\
m^{\dagger}=u M^{\dagger}+v M, \\
u^{2}-v^{2}=1 .
\end{gathered}
$$

For our problem at hand, we can generalize (55) by introducing two sets of operators $m_{n}$ and $m_{n}^{\dagger}(n=0,1, \ldots)$, which are the counterparts of the $m$ and $m^{\dagger}$ in (55), as the linear combination of the $M$ and $M^{\dagger}$ operators [59]:

$$
\begin{aligned}
m_{n}(P) & =\int_{-\infty}^{+\infty} \frac{d q}{\sqrt{2 \pi|P|}}\left[M(q-P, q) \varphi_{n}^{+}(q, P)+M^{\dagger}(q, q-P) \varphi_{n}^{-}(q, P)\right] \\
m_{n}^{\dagger}(P) & =\int_{-\infty}^{+\infty} \frac{d q}{\sqrt{2 \pi|P|}}\left[M^{\dagger}(q-P, q) \varphi_{n}^{+}(q, P)+M(q, q-P) \varphi_{n}^{-}(q, P)\right] \\
M(q-P, q) & =\sqrt{\frac{2 \pi}{|P|}} \sum_{n=0}^{\infty}\left[m_{n}(P) \varphi_{n}^{+}(q, P)-m_{n}^{\dagger}(-P) \varphi_{n}^{-}(q-P,-P)\right] \\
M^{\dagger}(q-P, q) & =\sqrt{\frac{2 \pi}{|P|}} \sum_{n=0}^{\infty}\left[m_{n}^{\dagger}(P) \varphi_{n}^{+}(q, P)-m_{n}(-P) \varphi_{n}^{-}(q-P,-P)\right],
\end{aligned}
$$

where $m_{n}(P)$ and $m_{n}^{\dagger}(P)$ will be interpreted as the annihilation and creation operators for the $n$th mesonic state carrying spatial momentum $P$. The functions $\varphi_{n}^{+}(q, P)$ and $\varphi_{n}^{-}(q, P)$ play the role of Bogoliubov coefficients $u$ and $v$ in (55a).

Similar to (22) in the LF case, here we again postulate that the mesonic annihilation and creation operators, $m_{n}$ and $m_{n}^{\dagger}$, obey the canonical commutation relations:

$$
\begin{aligned}
& {\left[m_{n}(P), m_{m}^{\dagger}\left(P^{\prime}\right)\right]=2 \pi \delta_{n m} \delta\left(P-P^{\prime}\right),} \\
& {\left[m_{n}(P), m_{m}\left(P^{\prime}\right)\right]=\left[m_{n}^{\dagger}(P), m_{m}^{\dagger}\left(P^{\prime}\right)\right]=0 .}
\end{aligned}
$$

In order to satisfy these commutation relations, the Bogoliubov functions $\varphi_{ \pm}^{n}$ must obey the following orthogonality and completeness conditions?

\footnotetext{
${ }^{7}$ We stress that our normalization conditions differ from those in [59] by a factor of $|P|$, because we demand that $\varphi_{+}^{n}(x P, P)$ remains dimensionless in conformity to the 't Hooft wave function $\phi(x)$, which turns out to be particularly convenient in comparing quasi- and light-cone distributions. Nevertheless, by adopting this convention, we are no longer capable of studying the bound-state solutions in the rest frame $(P=0)$ as was done in [62].
} 


$$
\begin{aligned}
\int_{-\infty}^{+\infty} d p\left[\varphi_{+}^{n}(p, P) \varphi_{+}^{m}(p, P)-\varphi_{-}^{n}(p, P) \varphi_{-}^{m}(p, P)\right] & =|P| \delta^{n m} \\
\int_{-\infty}^{+\infty} d p\left[\varphi_{+}^{n}(p, P) \varphi_{-}^{m}(p-P,-P)-\varphi_{-}^{n}(p, P) \varphi_{+}^{m}(p-P,-P)\right] & =0, \\
\sum_{n=0}^{\infty}\left[\varphi_{+}^{n}(p, P) \varphi_{+}^{n}(q, P)-\varphi_{-}^{n}(p-P,-P) \varphi_{-}^{n}(q-P,-P)\right] & =|P| \delta(p-q) \\
\sum_{n=0}^{\infty}\left[\varphi_{+}^{n}(p, P) \varphi_{-}^{n}(q, P)-\varphi_{-}^{n}(p-P,-P) \varphi_{+}^{n}(q-P,-P)\right] & =0 .
\end{aligned}
$$

Note the relative minus sign in the last two equations reflects the characteristic of the Bogoliubov transformation, as specified in (55c) [59].

We wish to diagonalize the axial-gauge Hamiltonian in the basis of $m_{n}$ and $m_{n}^{\dagger}$ operators. Applying the Bogoliubov transformation (56) into (52), we aim to obtain the intended form

$$
H=H_{0}^{\prime}+\int \frac{d P}{2 \pi} \sum_{n} P_{n}^{0} m_{n}^{\dagger}(P) m_{n}(P)+\mathcal{O}(1 / \sqrt{N}),
$$

where $P_{n}^{0}=\sqrt{M_{n}^{2}+P^{2}}$, with $M_{n}$ the mass of the $n$th mesonic state. The interaction terms involving three or more mesons are suppressed by powers of $1 / \sqrt{N}$, which is completely immaterial to the theme of this work, so will be neglected.

The shifted vacuum energy in (59) is given by

$$
\begin{aligned}
H_{0}^{\prime}= & H_{0}+\sum_{n} \int d z \int \frac{d P d p}{2 \pi|P|} \\
& \times\left\{[\tilde{E}(p)+\tilde{E}(P-p)] \varphi_{-}^{n}(p, P) \varphi_{-}^{n}(p, P)\right. \\
& -\lambda \int_{-\infty}^{+\infty} \frac{d k}{(p-k)^{2}} \Theta(|p-k|-\rho) \\
& \times\left[C(p, k, P) \varphi_{-}^{n}(p, P) \varphi_{-}^{n}(p, P)\right. \\
& \left.\left.-S(p, k, P)\left(\varphi_{+}^{n}(p, P) \varphi_{-}^{n}(k, P)+\varphi_{-}^{n}(p, P) \varphi_{+}^{n}(k, P)\right)\right]\right\},
\end{aligned}
$$

where $H_{0}$ is given in (40a).

We define the meson vacuum state $|\Omega\rangle$ by the condition

$$
m_{n}(P)|\Omega\rangle=0,
$$

for all $n$ and $P$. Consequently, a single meson state can be constructed via

$$
\left|P_{n}^{0}, P\right\rangle=\sqrt{2 P_{n}^{0}} m_{n}^{\dagger}(P)|\Omega\rangle
$$

Note the true vacuum state $|\Omega\rangle$ is highly nontrivial in the equal-time quantization. This is clearly illustrated by $(56 \mathrm{~b})$, according to which there are actually two very different mechanisms to create a meson. First is by creating a pair of quark and antiquark, no matter the vacuum is trivial or not. The other mechanism is by removing a pair of quark and antiquark from the vacuum. This is possible only if a flurry of correlated multi quark-antiquark constantly popping out of the vacuum, plausibly a consequence of the nonzero quark condensate. $^{8}$

After applying the Bogoliubov transformation to (52), in order to achieve the diagonalized form of (59), we have to enforce the coefficients of operators $m_{n}^{\dagger} m_{r} \quad(n \neq r)$, $m_{n}^{\dagger} m_{r}^{\dagger}+m_{n} m_{r}$ to vanish. After some algebra, we end up with the two following equations:

$$
\begin{aligned}
& {[\tilde{E}(p)}\left.\left.+\tilde{E}(P-p) \mp P_{n}^{0}\right)\right] \varphi_{n}^{ \pm}(p, P) \\
&= \lambda \int_{-\infty}^{+\infty} \frac{d k}{(p-k)^{2}} \Theta(|p-k|-\rho) \\
& \quad \times\left[C(p, k, P) \varphi_{n}^{ \pm}(k, P)-S(p, k, P) \varphi_{n}^{\mp}(k, P)\right] .
\end{aligned}
$$

This pair of equations is not particularly convenient to use, since both $\tilde{E}$ and the integrals are sensitive to the IR cutoff $\rho$. Miraculously, one can absorb the divergent $\frac{\lambda}{\rho}$ piece in $\tilde{E}$ into the cutoff-dependent integral, so that the modified integral becomes regular in the $\rho \rightarrow 0+$ limit. After some manipulation, the axial-gauge bound state equations (63) can be rewritten as

\footnotetext{
${ }^{8}$ Note the true vacuum $|\Omega\rangle$ is different from the quark vacuum $|0\rangle$ defined in (38). It is supposed that they are connected by a unitary operator $S$ [64], whose explicit form is unknown yet. In the following sections, we will always use the true ("mesonic") vacuum when computing QCD matrix elements.
} 


$$
\begin{aligned}
& \left(E(p)+E(P-p)-P^{0}\right) \varphi_{+}(p, P)=\lambda f_{-\infty}^{\infty} \frac{d k}{(p-k)^{2}}\left[C(p, k, P) \varphi_{+}(k, P)-S(p, k, P) \varphi_{-}(k, P)\right], \\
& \left(E(p)+E(P-p)+P^{0}\right) \varphi_{-}(p, P)=\lambda f_{-\infty}^{\infty} \frac{d k}{(p-k)^{2}}\left[C(p, k, P) \varphi_{-}(k, P)-S(p, k, P) \varphi_{+}(k, P)\right],
\end{aligned}
$$

where $E(p)$ is the regularized dressed quark energy defined in (48). Note that here we recover the principal value prescription as introduced in (28). ${ }^{9}$

Equations (64) are the very bound-state equations in $\mathrm{QCD}_{2}$ in axial gauge, first derived by Bars and Green back in 1978 [36]. For this reason, these equations will be referred to as Bars-Green equations. Consequently, the Bogoliubov-type functions $\varphi_{ \pm}^{n}$ can now be interpreted as the bound-state wave functions, or simply called BarsGreen wave functions.

A crucial feature of $\mathrm{QCD}_{2}$ in axial gauge is that, it preserves Poincaré invariance in physical sector in a highly nontrivial way. Notice the dispersive law for a colored object like dressed quark, which is encoded in (47) and (48), is clearly not Lorentz covariant. However, as far as the color-singlet meson is concerned, one is ensured to recover the standard dispersion relation dictated by special relativity. Specifically speaking, irrespective of the Lorentz frame where the Bars-Green equations (64) are tackled, one always ends up with the identical meson spectra, where the energy of the $n$th mesonic state is always found to satisfy $P_{n}^{0}=\sqrt{M_{n}^{2}+P^{2}}$. It is important to emphasize that, in order to preserves Poincaré invariance, the Bogoliubov angle $\theta(p)$ and the backward-motion component of BarsGreen wave functions, $\phi_{-}^{n}$, appear to play a indispensable role in (64). Thus, the 't Hooft model in axial gauge represents a rare example that one knows exactly how to consistently boost a relativistic bound-state wave function in the equal-time quantization.

A specific consequence of Poincaré invariance is that, when the meson is viewed the IMF, that is, in the $P \rightarrow \infty$ limit, one would still obtain the identical mesonic mass spectra. In this specific Lorentz frame, $\theta(p) \rightarrow \frac{\pi}{2}$ as $p \rightarrow \infty$, and the $C, S$ functions in (53) reduce to simple step functions, one can show that the Bars-Green equations simply reduce to the 't Hooft equation. Consequently, in the IMF, the $\phi_{+}^{n}$ component of the Bars-Green wave function reduces to the 't Hooft light-cone wave functions, and the $\phi_{-}^{n}$ component fades away at a rate $\propto 1 / P_{z}^{2}[40]$.

\footnotetext{
${ }^{9}$ One certainly can also use the equivalent PV prescriptions as specified in (29). Another practically useful prescription is the subtraction scheme [62], that is, for a test function $f(y)$ which is regular at $y=x$, one has $f d y \frac{f(y)}{(x-y)^{2}} \equiv \int d y \frac{1}{(x-y)^{2}}[f(y)-f(x)-$ $\left.(y-x) f^{\prime}(x)\right]$.
}

The first numerical solution of Bars-Green equations was conducted by $\mathrm{Li}$ and collaborators in the late $1980 \mathrm{~s}$, yet only for stationary $\left(P_{z}=0\right)$ mesons [62]. Very recently, the Bars-Green equations (64), for the first time, were solved numerically for an arbitrary moving frame for a variety of quark mass [40], thus explicitly establishing the Poincaré invariance of the 't Hooft model in axial gauge. In particular, the authors of [40] concretely observe the tendency that, when the meson gets more and more boosted, the $\phi_{+}$component does converge to the 't Hooft wave function, while the $\phi_{-}$component quickly vanishes. Moreover, some other physical quantities, such as the quark condensate and meson decay constant, were also numerically investigated in different moving frames and prove to be Lorentz invariant [40]. These studies unequivocally confirm the key role played by the chiral angle $\theta(p)$ and $\phi_{-}^{n}$ to preserve the Poincare invariance.

\section{BUILDING PDF AND QUASI-PDF OUT OF BOUND-STATE WAVE FUNCTIONS}

Parton distribution functions (PDFs) count essentially the number density of a specific species of partons inside a parent hadron that carries a specific momentum fraction of the hadron, $x$. It is not an exaggeration to say that they form the most indispensable inputs for yielding predictions for any high-energy collision experiments involving hadron beams. In this section, starting from the operator definitions of light-cone PDFs and quasiPDFs, we are going to reexpress them in terms of the light-cone wave functions and Bars-Green wave functions for the $\mathrm{QCD}_{2}$ in the large- $N$ limit. We will also see that the Bogoliubov angle $\theta(p)$ will explicitly enter the expression for the quasi-PDFs. This may offer some useful insight on the nature of quasi-PDFs in $3+1$ dimensional QCD. In this section, we assume the meson is moving along the positive $\hat{z}$ axis, so $P>0$.

\section{A. Light-cone PDF}

Collins and Soper have given a gauge-invariant operator definition for the PDFs [65]. One can readily adapt their definition to $\mathrm{QCD}_{2}$. According to [65], the quark light-cone $\mathrm{PDF}$ in $\mathrm{QCD}_{2}$ is defined as the nonlocal light-cone correlators sandwiched between two equal-momentum mesons, which are the $n$th mesonic states carrying the light-cone momentum $P^{+}$: 


$$
\begin{aligned}
q_{n}(x)= & \int_{-\infty}^{+\infty} \frac{d \xi^{-}}{4 \pi} e^{-i x P^{+} \xi^{-}} \\
& \times\left\langle P_{n}^{-}, P^{+}\left|\bar{\psi}\left(\xi^{-}\right) \gamma^{+} \mathcal{W}\left[\xi^{-}, 0\right] \psi(0)\right| P_{n}^{-}, P^{+}\right\rangle_{C},
\end{aligned}
$$

where $P_{n}^{-}=M_{n}^{2} /\left(2 P^{+}\right)$, with $M_{n}$ the meson mass of the $n$-th state. Here $\psi$ denotes quark Dirac field, and

$$
\mathcal{W}\left[\xi^{-}, 0\right]=\mathcal{P}\left[\exp \left(-i g_{s} \int_{0}^{\xi^{-}} d \eta^{-} A^{+}\left(\eta^{-}\right)\right)\right]
$$

is the gauge link connecting the two quark fields, inserted to ensure the gauge invariance of the PDF. $x=k^{+} / P^{+}$is the light-cone momentum fraction carried by the parton with respect to that of the meson. By construction, the lightcone PDF in (65) is boost invariant along the $z$ direction. The subscript $C$ in (65) indicates the disconnected contribution such as

$$
\begin{aligned}
& \left\langle P_{n}^{-}, P^{+} \mid P_{n}^{-}, P^{+}\right\rangle \int_{-\infty}^{+\infty} \frac{d \xi^{-}}{4 \pi} e^{-i x P^{+} \xi^{-}} \\
& \quad \times\left\langle 0\left|\bar{\psi}\left(\xi^{-}\right) \gamma^{+} \mathcal{W}\left[\xi^{-}, 0\right] \psi(0)\right| 0\right\rangle
\end{aligned}
$$

should be discarded when calculating the forward matrix element (65) [66].

PDF has a most transparent probabilistic interpretation in the light-front quantization framework [66]. Moreover, being a gauge-invariant quantity, the simplest way to proceed is to impose the light-cone gauge $A_{-}=0$ in (65), so that the gauge link can be dropped. For simplicity, we will adopt the LF quantization and light-cone gauge in $\mathrm{QCD}_{2}$, as was comprehensively described in Sec. III.

The presence of $\gamma^{+}$in (65) implies that only the $\psi_{R}$ component ("good" component) of the Dirac field $\psi$ is projected out. Applying the Fourier expansion of the $\psi_{R}$ as in (13), and replacing the meson state by acting $m_{n}^{\dagger}\left(P^{+}\right)$on the vacuum, and the light-cone PDF in (65) then becomes

$$
\begin{aligned}
q_{n}(x)= & \int_{-\infty}^{+\infty} \frac{d \xi^{-}}{4 \pi} e^{-i x P^{+} \xi^{-}} \iint \frac{d k_{1}^{+} d k_{2}^{+}}{4 \pi^{2}} \\
& \times\langle 0| m_{n}\left(P^{+}\right) \sqrt{2 P^{+}}\left[b^{\dagger}\left(k_{1}^{+}\right) e^{i k_{1}^{+} \xi^{-}}\right. \\
& \left.+d\left(k_{1}^{+}\right) e^{-i k_{1}^{+} \xi^{-}}\right]\left[b\left(k_{2}^{+}\right)+d^{\dagger}\left(k_{2}^{+}\right)\right] \sqrt{2 P^{+}} m_{n}^{\dagger}\left(P^{+}\right)|0\rangle_{C} .
\end{aligned}
$$

Replacing the combinations $b^{\dagger}\left(k_{1}^{+}\right) b\left(k_{2}^{+}\right), b^{\dagger}\left(k_{1}^{+}\right) d^{\dagger}\left(k_{2}^{+}\right)$, $d\left(k_{1}^{+}\right) b\left(k_{2}^{+}\right)$, and $d\left(k_{1}^{+}\right) d^{\dagger}\left(k_{2}^{+}\right)$by the bosonic operators $B$, $M^{\dagger}, M$, and $D$ as in (15) correspondingly, and rewriting $B$ and $D$ in terms of $M, M^{\dagger}$ according to (19), then eliminating $M, M^{\dagger}$ in favor of $m_{n}$ or $m_{n}^{\dagger}$ in line with (21), we end up with the vacuum matrix element of the product of a string of mesonic creation and annihilation operators. Discarding the disconnected piece (67), which arises from the commutator between $m_{n}$ and $m_{n}^{\dagger}$, we obtain the intended LC PDF for the $n$th mesonic state,

$$
q_{n}(x)=\varphi_{n}(x)^{2},
$$

where $\varphi_{n}(x)$ is the 't Hooft light-cone wave function associated with the $n$th mesonic state. This result confirms what was obtained in [35] using simpler method. Hearteningly, the light-cone PDF in 't Hooft model looks exceedingly simple.

It is also straightforward to account for the antiquark distribution, by extending the support of $x$ in (69) from $0 \leq x \leq 1$ to $-1 \leq x \leq 1$,

$$
q_{n}(x)=\epsilon(x) \varphi_{n}(|x|)^{2},
$$

with the sign function $\epsilon(x)$ equal to 1 for positive $x$, and equal to -1 for negative $x$. Since we are considering only the flavor-neutral meson, the antiquark PDF is obviously identical to the quark PDF.

\section{B. Quasi-PDF}

The quasi-PDF was recently introduced by $\mathrm{Ji}$ as a proxy to facilitate the extraction of the light-cone PDF from Euclidean lattice QCD [6,7]. It is defined as the equal-time spatially nonlocal correlation functions sandwiched between two equal-momentum hadrons. Although the quasi-PDF is obviously not boost-invariant, its profile is expected to converge to the light-cone PDF in the IMF. Analogous to the definition of the quasi-PDF in realistic QCD [6], the quasi-PDF in $\mathrm{QCD}_{2}$ can be defined as the following forward matrix element, with the external hadron taken to be the $n$-th mesonic state with the spatial momentum $P$ :

$\tilde{q}_{n}(x, P)=\int_{-\infty}^{+\infty} \frac{d z}{4 \pi} e^{i x P z}\left\langle P_{n}^{0}, P\left|\bar{\psi}(z) \gamma^{z} \mathcal{W}[z, 0] \psi(0)\right| P_{n}^{0}, P\right\rangle_{C}$,

where $P_{n}^{0}=\sqrt{P^{2}+M_{n}^{2}}, x=k / P$ is the spatial momentum fraction carried by the parton with respect to that of the meson. Unlike the light-cone PDF, the range of $x$ is unconstrained, $-\infty<x<\infty$. The spacelike gauge link

$$
\mathcal{W}[z, 0]=\mathcal{P}\left[\exp \left(-i g_{s} \int_{0}^{z} d z^{\prime} A^{z}\left(z^{\prime}\right)\right)\right]
$$

has been inserted in (71) to ensure gauge invariance of the quasi-PDF.

Similar to (65), the subscript $C$ in (71) again indicates that only the connected contributions are included. Therefore, the disconnected piece,

$\left\langle P_{n}^{0}, P \mid P_{n}^{0}, P\right\rangle \int_{-\infty}^{+\infty} \frac{d z}{4 \pi} e^{i x P z}\left\langle\Omega\left|\bar{\psi}(z) \gamma^{z} \mathcal{W}[z, 0] \psi(0)\right| \Omega\right\rangle$

should be discarded when calculating the matrix element affiliated with the quasi-PDF. 
Since the quasi-PDF is time independent, it is natural to study its property in the equal-time quantization. Moreover, it is most convenient to compute the quasi-PDF in the axial gauge $A^{z}=0$ in (71), so that one can neglect the gauge link. For simplicity, in this subsection, we will stay with the equal-time quantization and work with axial gauge in $\mathrm{QCD}_{2}$, closely following the quantization procedure detailed in Sec. IV.

We proceed by the bosonization procedure similar to computing the light-cone PDF in Sec. VA. First replacing the meson state by acting $m_{n}^{\dagger}(P)$ on the true vacuum $|\Omega\rangle$, our task then becomes to compute the vacuum matrix element. Conducting the Fourier expansion of the Dirac field $\psi$ in accordance with (36), expressing the product of two quark annihilation and creation operators in terms of $B$, $M^{\dagger}, M$, and $D$ as introduced in (49), followed by rewriting $B, D$ as the convolution integral between $M, M^{\dagger}$ according to (51), then trading $M, M^{\dagger}$ for the meson annihilation and creation operators $m_{n}$ or $m_{n}^{\dagger}$ in line with (56), we end up with the vacuum matrix element of the product of a string of meson annihilation and creation operators. Repeatedly applying the commutation relations

$$
\begin{aligned}
\tilde{q}_{n}(x, P)= & \frac{P_{n}^{0}}{P} \sin \theta(x P)\left[\left(\varphi_{+}^{n}(x P, P)\right)^{2}+\left(\varphi_{-}^{n}(x P, P)\right)^{2}\right. \\
& \left.+\left(\varphi_{+}^{n}(-x P, P)\right)^{2}+\left(\varphi_{-}^{n}(-x P, P)\right)^{2}\right] .
\end{aligned}
$$

The explicit occurrences of the Bogliubov angle $\theta(p)$, and the backward-moving component of the Bars-Green wave functions $\varphi_{-}^{n}$, make the quasi-PDF a much more complicated object than the light-cone PDF. It is reassuring to see that, in the IMF, i.e., in the $P \rightarrow \infty$ limit, where $P_{n}^{0} \rightarrow P$, $\theta(x P) \rightarrow \frac{\pi}{2} \epsilon(x)$, and $\phi_{-}^{n}$ dies away, the quasi-PDF in (74) does recover the light-cone PDF in (69).

Equation (74) is one of the key achievements of this paper. We have successfully constructed the quasi-PDF in terms of the basic building block of $\mathrm{QCD}_{2}$ in axial gauge, the chiral angle and the Bars-Green wave functions. We are wondering whether this reduction pattern, at least to some extent, can also be carried over to the realistic QCD.

Charge conjugation symmetry imposes the following relation for the $\tilde{q}(x)$ :

$$
\tilde{q}(-x, P)=-\tilde{q}(x, P) .
$$

Reassuringly, the quasi-PDF as specified in (74), indeed obeys this relation.

It is worth mentioning here, the definition of quasi-PDF is by no means unique. In principle, one can construct an infinite number of gauge-invariant quasi-PDFs, all of which are equally legitimate provided that all of them can reduce to the light-cone PDF in IMF. It can be said that all the legal definitions of quasi-PDFs form a universality class [67]. In Appendix A, we will numerically compare two different definitions of quasi-PDF, the one just considered in this section, versus the other defined by replacing $\gamma^{z}$ in (71) with $\gamma^{0}$.

\section{BUILDING LCDA AND QUASI-DA OUT OF BOUND-STATE WAVE FUNCTIONS}

For hard exclusive reactions involving hadrons, it is the light-cone distribution amplitude (LCDA), rather than the PDF, that directly enters the QCD factorization theorem [68]. Therefore, LCDAs represent the fundamental nonperturbative inputs in order to describe the hard exclusive QCD processes.

Analogous to Sec. V, we will in this section express the LCDA and quasi-DA of a flavor-neutral meson in $\mathrm{QCD}_{2}$, in terms of its bound-state wave functions.

\section{A. LCDA}

In line with Refs. $[68,69]$, one defines the LCDA of a flavor-neutrual meson in $\mathrm{QCD}_{2}$ as

$$
\begin{aligned}
\Phi_{n}(x)= & \frac{1}{f^{(n)}} \int_{-\infty}^{+\infty} \frac{d \xi^{-}}{2 \pi} e^{-i\left(x-\frac{1}{2}\right) P^{+} \xi^{-}}\left\langle P_{n}^{-}, P^{+}\right| \bar{\psi}\left(\frac{\xi^{-}}{2}\right) \\
& \times \mathcal{W}\left[\frac{\xi^{-}}{2},-\frac{\xi^{-}}{2}\right] \gamma^{+} \gamma_{5} \psi\left(-\frac{\xi^{-}}{2}\right)|0\rangle,
\end{aligned}
$$

where $\mathcal{W}$ is the lightlike gauge link similar to what is introduced in (65). $f^{(n)}$ denotes the decay constant of the $n$th mesonic state, defined through $[40,51]$

$$
\left\langle n, P\left|\bar{\psi} \gamma^{\mu} \gamma^{5} \psi\right| \Omega\right\rangle=f^{(n)} \frac{P^{\mu}}{\sqrt{2 P^{0}}} .
$$

Analogous to Sec. VA, it is most transparent to study the LCDA in LF quantization supplemented with further imposing the light-cone gauge. The expressions of the meson decay constants are particularly simple in LF quantization [51],

$$
f^{(n)}= \begin{cases}\sqrt{\frac{N}{\pi}} \int_{0}^{1} d x \varphi_{n}(x) & \text { even } n, \\ 0 & \operatorname{odd} n,\end{cases}
$$

which are particularly simple. Due to the parity consideration, the decay constants of all the $n$-odd flavor-neutral mesons vanish. Therefore, we will concentrate on the LCDAs of those $n$-even mesonic states.

Following the essentially same bosonization techniques that lead to the light-cone PDF in Sec. VA, we find the LCDA for the $n$-even mesonic states to be

$$
\Phi_{2 n}(x)=\frac{1}{f^{(2 n)}} \sqrt{\frac{N}{\pi}} \varphi_{2 n}(x),
$$

which is simply proportional to the 't Hooft wave function. Since the decay constant scales as $\sqrt{N}$, the LCDAs thereby assume finite value in the $N \rightarrow \infty$ limit. As a matter of fact, 
the LCDAs are subject to the normalization condition by construction:

$$
\int_{0}^{1} d x \Phi_{2 n}(x)=1
$$

\section{B. Quasi-DA}

Analogous to the quasi-DA introduced in $\mathrm{QCD}_{4}[6,7]$, here we choose to define the quasi-DA in $\mathrm{QCD}_{2}$ in its canonical form,

$$
\begin{aligned}
\tilde{\Phi}_{n}(x, P)= & \frac{1}{f^{(n)}} \int_{-\infty}^{+\infty} \frac{d z}{2 \pi} e^{i\left(x-\frac{1}{2}\right) P z} \\
& \times\left\langle P_{n}^{0}, P\left|\bar{\psi}\left(\frac{z}{2}\right) \mathcal{W}\left[\frac{z}{2},-\frac{z}{2}\right] \gamma^{z} \gamma_{5} \psi\left(-\frac{z}{2}\right)\right| \Omega\right\rangle,
\end{aligned}
$$

where $\mathcal{W}$ is the spacelike gauge link as introduced in (71).
Employing essentially the same bosonization procedure, which leads to the analytic expression for quasi-PDF in Sec. V B, we finally find that the quasi-DAs of those $n$-even mesonic states can be formulated as

$$
\begin{aligned}
\tilde{\Phi}_{2 n}(x, P)= & \frac{1}{f^{(2 n)}} \sqrt{\frac{N}{\pi}} \sqrt{\frac{P^{0}}{P}} \sin \frac{\theta(x P)+\theta(P-x P)}{2} \\
& \times\left[\varphi_{+}^{2 n}(x P, P)+\varphi_{-}^{2 n}(x P, P)\right],
\end{aligned}
$$

where $\varphi_{ \pm}^{2 n}$ denote the Bars-Green wave functions associated with the $2 n$th excited mesonic state. The explicit form of the decay constant $f^{(n)}$ in axial gauge has also been worked out [40], which looks considerably more complicated than the LF quantization case:

$$
f^{(n)}= \begin{cases}\sqrt{\frac{N P^{0}}{\pi P}} \int_{-\infty}^{\infty} d x \sin \frac{\theta(x P)+\theta(P-x P)}{2}\left[\varphi_{+}^{n}(x P, P)+\varphi_{-}^{n}(x P, P)\right] & \text { even } n \\ 0 & \text { odd } n\end{cases}
$$

Note the Bars-Green wave functions and the Bogoliubov angle conspire in a nontrivial manner so that $f^{(n)}$ is independent of the Lorentz frame.

Comparing (82) and (83), one sees that, by construction the quasi-DAs also obey a very simple normalization condition:

$$
\int_{-\infty}^{\infty} d x \tilde{\Phi}_{2 n}(x, P)=1
$$

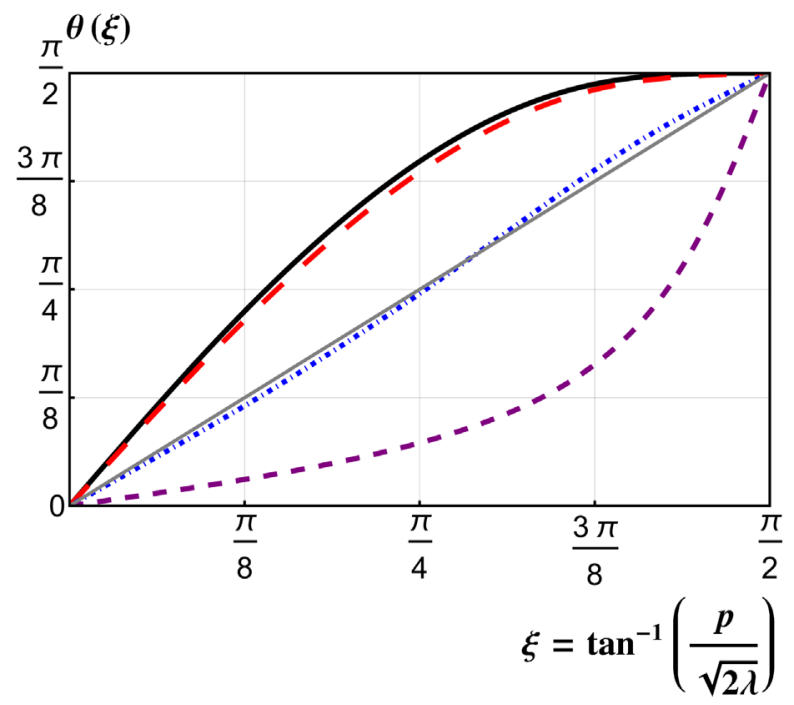

Reassuringly, in the IMF $(P \rightarrow \infty)$, one readily verifies that, the quasi-DA in (82) does recover the LCDA as given in (79).

\section{NUMERICAL RESULTS OF QUASI-PDF AND -DA}

Based on the analytic expressions for the light-cone and quasidistributions worked out in the preceding sections, we are going to present a comparative study for quasidistributions

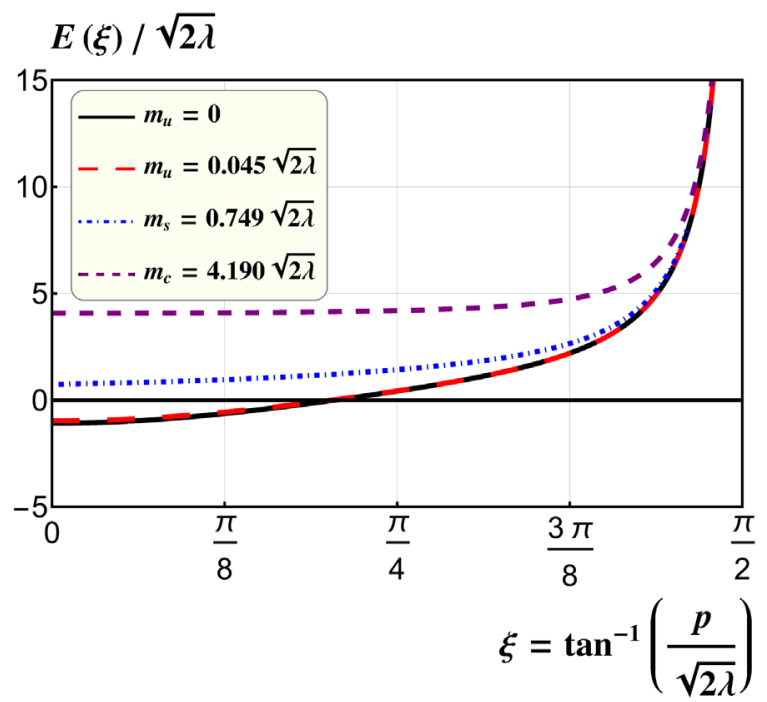

FIG. 1. Bogoliubov-chiral angle $\theta(p)$, and the regularized dressed quark energy, $E(p)$, as functions of $\xi=\tan ^{-1}\left(\frac{p}{\sqrt{2 \lambda}}\right)$, with different quark masses as specified in Table I. Most curves are taken from Ref. [40], except those for the charm quark. 
TABLE I. Quark masses and the corresponding meson mass spectra, where only the ground state and the first excited state are included. $^{10}$

\begin{tabular}{lcccc}
\hline \hline$m_{q}$ & $u / d$ & $u / d$ & $s$ & $c$ \\
\hline & 0 & 0.045 & 0.749 & 4.190 \\
$M_{n}$ & $\pi_{\chi}$ & $\pi$ & $s \bar{s}$ & $c \bar{c}$ \\
$n=0$ & 0 & 0.41 & 2.18 & 9.03 \\
$n=1$ & 2.43 & 2.50 & 3.72 & 10.08 \\
\hline \hline
\end{tabular}

and their light-cone counterparts in this section. We consider four types of lowest-lying flavor-neutral mesons: chiral (massless) pion $\left(\pi_{\chi}\right)$, physical pion $\pi$, a fictitious "strangeonium" $s \bar{s}$, and charmonium, varying the quark masses according to the recipe described in Ref. [40]. For the lightcone and quasi-PDFs, we also consider the first excited states associated with these four meson species.

The 't Hooft coupling $\lambda=0.18 / \pi \mathrm{GeV}^{2}$ is taken to coincide with the value of the string tension in realistic $\mathrm{QCD}_{4}$ [35]. The quark masses (in units of $\sqrt{2 \lambda}$ ) are tuned in such a way that the ground-state meson masses coincide with the realistic meson masses of $\pi_{\chi}, \pi$ and $c \bar{c}$, while the mass of the $s$ quark is determined by demanding that the Bogoliubov angle $\theta(p)$ as a function of $\xi=\tan ^{-1}(p / \sqrt{2 \lambda})$ is closest to a straight line [40]. The numerical solutions of the 't Hooft equation, mass-gap equation and Bars-Green equations have already been presented comprehensively in Ref. [40], and we refer the interested reader to that paper for technical details. Here we will directly present our numerical results. For the sake of clarity, the profiles of the Bogoliubov angle $\theta(p)$ and the dispersion relation $E(p)$, which are affiliated with the aforementioned quark masses, are depicted in Fig. 1.

The quark masses and the corresponding meson mass spectra (for simplicity, we only include the ground state $n=0$ and the first excited state $n=1$ ) are listed in Table I.

In light of the numerically available 't Hooft and BarsGreen wave functions [40], as well as (74) and (82), we calculate the quasidistributions of those mesons in several different reference frames. The light-cone distributions are also juxtaposed for comparison. The numerical results of light-cone and quasi-PDFs for lowest-lying mesons are shown in Fig. 2, and those for the first excited state in Fig. 3, while the numerical results for the LCDAs and quasi-DAs of the ground-state mesons are presented in Fig. 4.

From Figs. 2, 3, and 4, one clearly observes the general tendency, that irrespective of the meson species, the

\footnotetext{
${ }^{10}$ In Ref. [40], the charm quark mass is "erroneously" take to be $m_{c}=4.23 \sqrt{2 \lambda}$. In this work, we take $m_{c}$ to be $4.19 \sqrt{2 \lambda}$, which is tuned to reproduce the center-of-gravity mass of the lowest-lying charmonia, $M_{\text {C.O.G }}=\frac{1}{4} M_{\eta_{c}}+\frac{3}{4} M_{J / \psi}$, associated with the real world.
}

quasidistributions are indeed converging to their light-cone counterparts, as the meson gets more and more boosted.

An interesting observation is that, the quasidistributions of heavier mesons $(s \bar{s}, c \bar{c})$ appear to converge to the lightcone distributions at a faster pace than those of lighter mesons $\left(\pi_{\chi}, \pi\right)$. To quantify this assertion, let us introduce the ratio $r \equiv P_{n} / M_{n}$. For light mesons, as exemplified by the physical pion, even when boosted to $r=8$, there still exists considerable difference between the shapes of the light-cone and quasidistributions; on the other hand, heavy mesons tends to exhibit a rather different pattern. When $r=5$ for the $s \bar{s}$ meson, or when $r=2$ for the $c \bar{c}$ meson, ${ }^{11}$ the quasidistributions already coincide with the light-cone distributions to a decent degree.

The correlation between the convergence behavior of the quasidistributions under boost and the hadron species has already been noticed in lattice simulations in realistic $\mathrm{QCD}_{4}$ [18-22]. There it is found that, somewhat counterintuitively, the nucleon's quasi-PDF approaches its lightcone PDF at a much faster pace than the quasi DA of pion approaches its LCDA.

If it is not a sheer accident, it will be valuable if the 't Hooft model can offer some insight into unraveling this curious correlation pattern observed in real world.

We end this section by commenting on a simple fact concerning the first excited state. As can be seen in Fig. 3, one observes a zero at $x= \pm \frac{1}{2}$ for various light-cone PDFs in the $n=1$ state, but not for the corresponding quasi PDFs. This can be understood from the angle of the charge conjugation symmetry [40],

$$
\begin{aligned}
\varphi^{n}(1-x) & =(-1)^{n} \varphi^{n}(x), \\
\tilde{\varphi}_{ \pm}^{n}(1-x, P) & =(-1)^{n} \tilde{\varphi}_{ \pm}(x, P),
\end{aligned}
$$

which implies that the light-cone as well as Bars-Green wave functions of the $n$-odd states must have a zero at $x=\frac{1}{2}$. Nevertheless, as one can see from (74), due to the presence of $\sin \theta(x P)$ as well as the different arguments arising in four types of squared Bars-Green functions, the quasi-PDFs in finite reference frame no longer possess the simple odd symmetry under $x \leftrightarrow 1-x$.

\section{ONE-LOOP PERTURBATIVE CALCULATIONS FOR LIGHT-CONE AND QUASIDISTRIBUTIONS AND IR DIVERGENCES}

So far, we have been completely concentrating on the nonperturbative aspects of the light-cone and quasidistributions in $\mathrm{QCD}_{2}$. In this section, we will instead switch the gear, to address some theoretical issues within the confine of perturbation theory.

\footnotetext{
${ }^{11}$ We stop at $r=2$ for charmonium, mainly because the technical challenge about numerical instability of boosting a heavy meson quickly becomes insurmountable.
} 

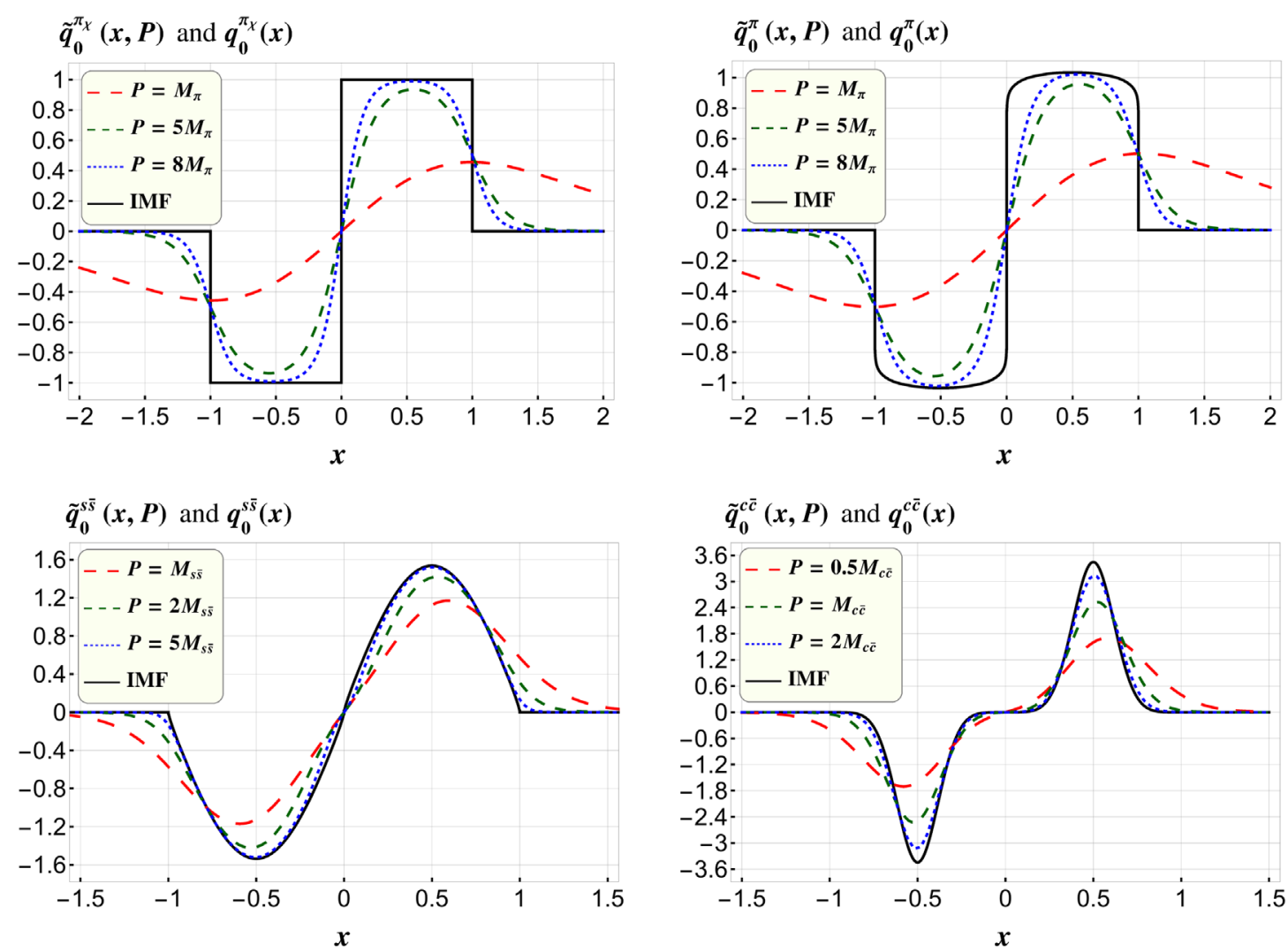

FIG. 2. Quark light-cone and quasi-PDFs for the chiral pion, physical pion, lowest-lying strangeonium and charmonium. The momentum of chiral pion is in unit of the physical pion mass.
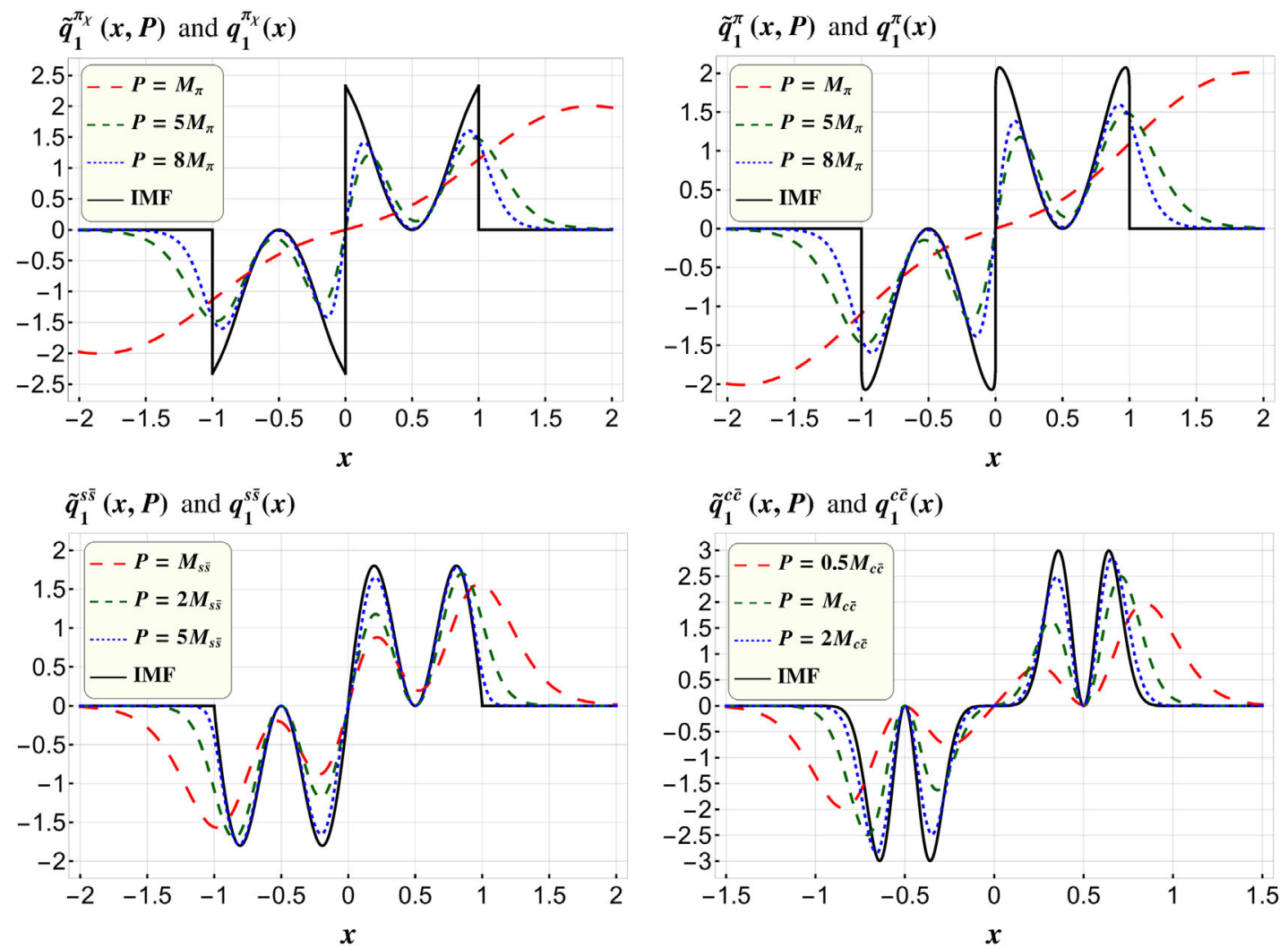

FIG. 3. Quark light-cone and quasi-PDFs for the 1st excited state corresponding to four different quark masses as specified in Table I. The meson momenta are in units of the ground-state mass for each quark specifies. 

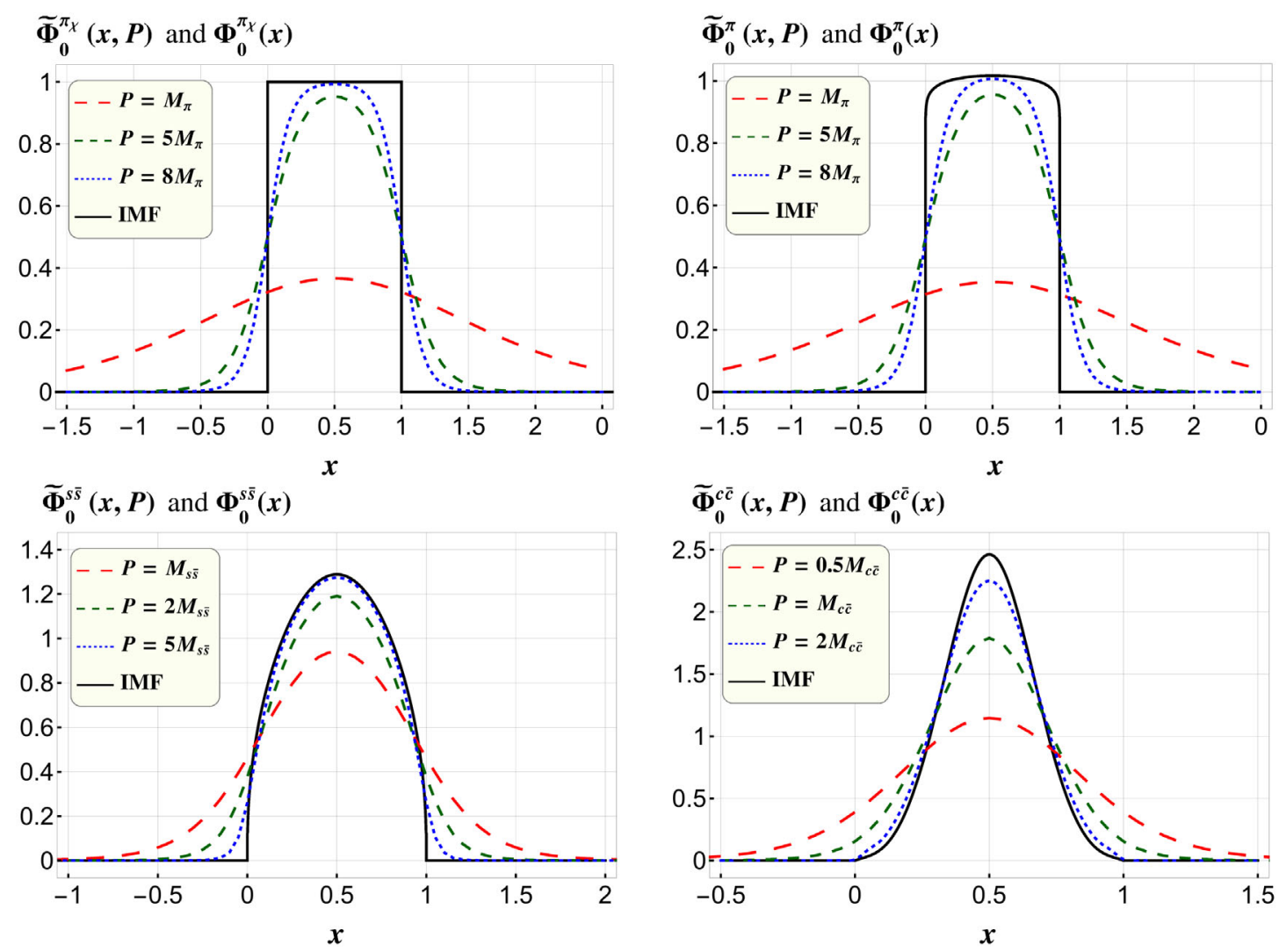

FIG. 4. LCDAs and quasi-DAs for the chiral pion, pion, lowest-lying strangeonium and charmonium. The momentum of the chiral pion is in units of the physical pion mass.

The key insight underlying LaMET in $\mathrm{QCD}_{4}$ is that quasidistributions exhibit the same IR behavior as their light-cone counterparts in the leading power of $1 / P^{z}[6]$. Because of this peculiar feature, there arises a factorization theorem that connects the quasi- and light-cone PDFs [6-8],

$\tilde{q}\left(x, P^{z}, \Lambda\right)=\int_{-1}^{1} \frac{d y}{|y|} Z\left(\frac{x}{y}, \frac{\Lambda}{P^{z}}, \frac{\mu}{P^{z}}\right) q(y, \mu)+\mathcal{O}\left(\frac{\Lambda_{\mathrm{QCD}}^{n}}{P_{z}^{n}}, \frac{M^{r}}{P_{z}^{r}}\right)$,

where $\Lambda$ represents a UV cutoff in the transverse momentum space associated with the quasi-PDF, and $\mu$ is the renormalization scale associated with the light-cone PDF. The neglected terms represent the higher-twist corrections. The factorization theorem (86) states that the $Z$ factor takes into account the difference between the UV regimes of the lightcone and quasi-PDFs, which is thus amenable to perturbation theory owing to the asymptotic freedom of QCD. Through the one-loop order, the $Z$ factor affiliated with the quark PDF can be expressed as

$Z\left(\xi, \frac{\Lambda}{P^{z}}, \frac{\mu}{P^{z}}\right)=\delta(\xi-1)+\frac{\alpha_{s}}{\pi} Z^{(1)}\left(\xi, \frac{\Lambda}{P^{z}}, \frac{\mu}{P^{z}}\right)+\cdots$

The order- $\alpha_{s}$ coefficient can be computed by the perturbative matching procedure,

$$
Z^{(1)}\left(\xi, \frac{\Lambda}{P^{z}}, \frac{\mu}{P^{z}}\right)=\tilde{q}^{(1)}\left(\xi, P^{z}, \Lambda\right)-q^{(1)}(\xi, \mu),
$$

where the physical hadron has been replaced by a single quark, $\tilde{q}^{(1)}$ and $q^{(1)}$ signify the corresponding quasi- and lightcone PDF associated with this "fictitious" hadron, accurate to the order- $\alpha_{s}$. In four spacetime dimensions, due to the severe UV divergence emerging from the transverse momentum integration, the limit of $P^{z} \rightarrow \infty$ and $\Lambda \rightarrow \infty$ generally do not commute [8]. It is this very noncommutativity that leads to a nontrivial matching factor in realistic QCD.

In this section, we will calculate the one-loop corrections to the light-cone and quasidistributions in $d=2$ spacetime dimensions. The major motif of such computation is to verify one of the backbone of LaMET, that quasi- and lightcone distributions indeed possess the same IR behavior in the leading order in $1 / P^{z}$, even in $\mathrm{QCD}_{2}$. Recall that the gauge coupling in $\mathrm{QCD}_{2}$ carries a positive mass dimension, thereby the 't Hooft model is a superrenormalizable theory. Therefore, the (almost) absence of UV divergences in loop diagrams ${ }^{12}$ nullifies the aforementioned noncommutativity;

\footnotetext{
${ }^{12}$ One exception is the perturbative correction to the quark condensate $\langle\bar{\psi} \psi\rangle$ in $\mathrm{QCD}_{2}$, which receives a logarithmic UV divergence from the one-loop tadpole diagram, and can be eliminated through additive renormalization [70].
} 
thus, we do not expect a nontrivial $Z$ factor to arise. On the other hand, $\mathrm{QCD}_{2}$ has much more severe IR divergences than its four-dimensional cousin, so it is interesting to explicitly examine the IR behavior of light-cone and quasidistributions.

At first sight, it may appear attractive to utilize dimensional regularization (DR) to regularize the IR divergence. Nevertheless, apart from automatically preserving Lorentz and gauge invariance, this popular regularization scheme is not suited for our purpose. First, we will encounter severe power IR divergences, which are simply absent in DR, but are actually what we desire to see. More importantly, DR in $1+1$-dimensional theory has some intrinsic drawback. When working in $2-2 \epsilon$ dimensions, we have artificially introduced some fictitious transverse degrees of freedom, which might lead to some pathological behavior when taking the $\epsilon \rightarrow 0$ limit in the end.

In formulating the bound-state equation in the Hamiltonian approach in Secs. III and IV, we have used an infrared momentum cutoff to regularize the IR divergence. In this section, we will again employ this "physical" IR cutoff, which turns out to be convenient and less confusing. It is worth mentioning that the large- $N$ limit is no longer required in this section.

\section{A. Light-cone and quasi-PDFs to one-loop order}

In computing the quark light-cone and quasi-PDFs, we replace a physical meson by a single quark. For technical simplicity, in this subsection, we will no longer stay with the noncovariant gauge, rather conduct all the calculation in Feynman gauge. The one-loop Feynman diagrams for quark PDF are shown in Fig. 5.

At this stage, we will treat the one-loop corrections for light-cone and quasi-PDF in a unified manner. Following the Feynman rules for PDF (quasi-PDF) and gluon-gaugue link interaction term in Ref. [66], the contributions from real correction diagrams are $[10,71]$

$$
\begin{aligned}
\mathcal{Q}_{a}^{R}(x, n) & =\frac{-i g_{s}^{2}}{2} \int \frac{d^{2} k}{(2 \pi)^{2}} \frac{\bar{u}(P) \gamma^{\mu}(k+m) \not h(k+m) \gamma_{\mu} u(P)}{\left(k^{2}-m^{2}+i \epsilon\right)^{2}\left[(P-k)^{2}+i \epsilon\right]} \delta(n \cdot k-x n \cdot P), \\
\mathcal{Q}_{b+c}^{R}(x, n) & =-i g_{s}^{2} \int \frac{d^{2} k}{(2 \pi)^{2}} \frac{\bar{u}(P) \not h(k+m) \not h u(P)}{\left(k^{2}-m^{2}+i \epsilon\right)\left[(P-k)^{2}+i \epsilon\right]} \frac{\delta(n \cdot k-x n \cdot P)}{n \cdot(P-k)}, \\
\mathcal{Q}_{d}^{R}(x, n) & =\frac{-i g_{s}^{2}}{2} \int \frac{d^{2} k}{(2 \pi)^{2}} \frac{n^{2} \bar{u}(P) h u(P)}{(P-k)^{2}+i \epsilon} \frac{\delta(n \cdot k-x n \cdot P)}{[n \cdot(P-k)]^{2}},
\end{aligned}
$$

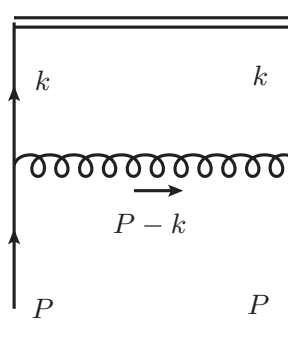

$R_{a}$

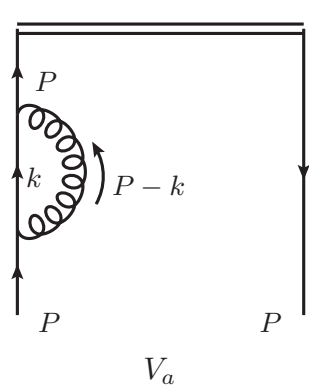

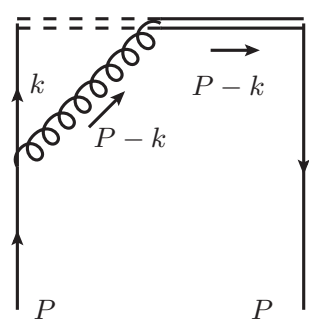

$R_{b}$

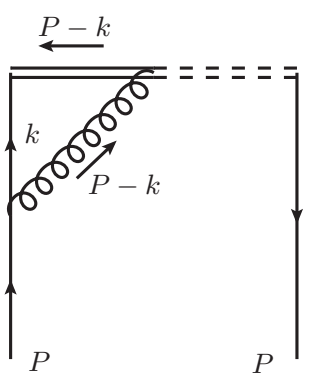

$V_{b}$

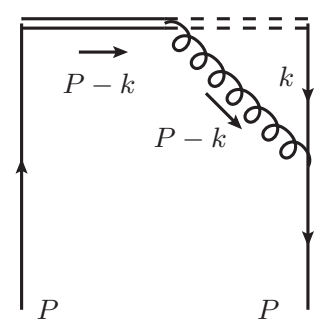

$R_{c}$

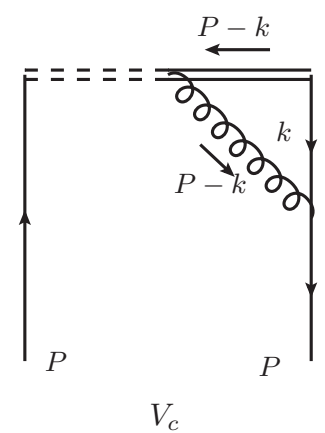

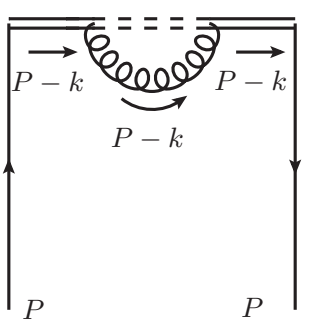

$R_{d}$

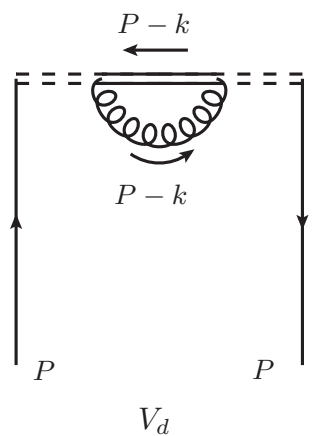

FIG. 5. One-loop Feynman diagrams for quark light-cone and quasi-PDF. The upper row corresponds to the real corrections $\mathcal{Q}^{R}$, while the lower row corresponds to the virtual corrections $\mathcal{Q}^{V}$ which are proportional to $\delta(x-1)$. The Feynman diagrams show the one-loop corrections to the process of a quark with momentum $P$ splitting to a quark with momentum $k\left(R_{a}\right)$, the quark interacting with gauge link though a gluon exchange $\left(R_{b, c}, V_{b, c}\right)$, the quark's self-energy $\left(V_{a}\right)$ and gauge link's self-interaction $\left(R_{d}, V_{d}\right)$. The double line represents the gauge link in PDF and quasi-PDF definition in (65) and (71), while the dashed double line represents the gauge link on which no net momentum flows. For simplicity, we have also omitted the wave function renormalization on the quark leg in the final state. 
and the contributions from virtual correction diagrams are $[10,71]$

$$
\begin{aligned}
\mathcal{Q}_{a}^{V}(x, n) & =\delta Z_{F} \delta(1-x)=-\left.\delta(1-x) \frac{d \Sigma(P)}{d \not P}\right|_{\not p=m}, \\
\mathcal{Q}_{b+c}^{V}(x, n) & =-\delta(x-1) \int d x \mathcal{Q}_{b+c}^{R}(x, n), \\
\mathcal{Q}_{d}^{V}(x, n) & =-\delta(x-1) \int d x \mathcal{Q}_{d}^{R}(x, n),
\end{aligned}
$$

where $\Sigma(P)$ represents the quark self-energy, and $Z_{F}$ denotes the quark wave function renormalization constant.
The above expressions in (89) and (90) can be adapted to both light-cone or quasi-PDF, depending on the specific choice of the reference vector $n^{\mu}$. For the former, $n^{\mu}$ is chosen to be the null vector $n^{\mu}=n_{\mathrm{LC}}^{\mu}$ such that $n_{\mathrm{LC}}^{2}=0$, $k \cdot n_{\mathrm{LC}}=k^{+}$, and $\gamma \cdot n_{\mathrm{LC}}=\gamma^{+}$; while for the latter, the reference vector is taken to be $n_{z}^{\mu}$, such that $n_{z}^{2}=-1$, $k \cdot n_{z}=k^{z}$, and $\gamma \cdot n_{z}=\gamma^{z}$.

To compute the one-loop corrections to the light-cone $\mathrm{PDF}$, it is convenient to switch to the light-cone coordinate, so one can write $P \cdot k=P^{+} k^{-}+P^{-} k^{+}$. The integration over $k^{+}$can be trivially carried out using the $\delta$ function, while the $k^{-}$integration is performed via the method of residue. Summing all the real correction diagrams, we find a null result,

$q^{R}(x)=\mathcal{Q}_{a}^{R}\left(x, n_{\mathrm{LC}}\right)+\mathcal{Q}_{b+c}^{R}\left(x, n_{\mathrm{LC}}\right)+\mathcal{Q}_{d}^{R}\left(x, n_{\mathrm{LC}}\right)= \begin{cases}\left(\frac{-x g_{s}^{2} C_{F}}{\pi m^{2}(1-x)^{3}}\right)_{a}+\left(\frac{x g_{s}^{2} C_{F}}{\pi m^{2}(1-x)^{3}}\right)_{b+c}+(0)_{d} \quad 0<x<1=0 \\ 0 & \text { Otherwise }\end{cases}$

where the subscript $a, b+c, d$ denote the contributions from $R^{a}, R^{b}+R^{c}$, and $R^{d}$ in Fig. 5, respectively.

In computing the virtual corrections (90), we have employed the momentum fraction $\eta$ as an IR regulator, imposed on the $k^{+}$integration,

$$
\int_{0}^{P^{+}} d k^{+} \Rightarrow P^{+} \int_{0}^{1} d x \Theta(1-x-\eta)
$$

where $x=k^{+} / P^{+}$and $\eta \rightarrow 0^{+}$.

The sum of the virtual diagrams also vanishes:

$$
q^{V}(x)=\mathcal{Q}^{V}\left(x, n_{\mathrm{LC}}\right)=\left[\delta(x-1) \frac{g_{s}^{2} C_{F}}{2 \pi m^{2}}\left(\frac{1}{\eta^{2}}-\frac{2}{\eta}+1\right)\right]_{a}+\left[-\delta(x-1) \frac{g_{s}^{2} C_{F}}{2 \pi m^{2}}\left(\frac{1}{\eta^{2}}-\frac{2}{\eta}+1\right)\right]_{b+c}+(0)_{d}=0 .
$$

Piecing all terms together, the light-cone PDF at one-loop level is

$$
q(x)=q^{R}(x)+q^{V}(x)=0 .
$$

The vanishing light-cone PDF in two dimensions is not surprising. The one-loop quark PDF can be interpreted as the probability of a parent quark splitting into a daughter quark plus a on-shell gluon. However, there is no physical gluon in two dimensions because the lacking of transverse degree of freedom. Consequently, the one-loop light-cone PDF (splitting function) vanishes.

For the quasi-PDF, we stay with the ordinary coordinates and the reference two-vector $n$ is chosen as the spacelike unit vector $n_{z}$. For the real correction diagrams, first performing the $k^{z}$ integration using the $\delta$ function, then integrating over $k^{0}$ via the method of residues, we obtain 


$$
\begin{aligned}
& \tilde{q}_{a}^{R}\left(x, P^{z}\right)= \mathcal{Q}_{a}^{R}\left(x, n_{z}\right)=\frac{g_{s}^{2} C_{F} m^{2}}{4 \pi}\left\{\frac{x P^{z}\left[2 P^{0} \sqrt{m^{2}+x^{2} P_{z}^{2}}+2 m^{2}+x(x+1) P_{z}^{2}\right]}{\left(m^{2}+x^{2} P_{z}^{2}\right)^{3 / 2}\left(P^{0} \sqrt{m^{2}+x^{2} P_{z}^{2}}+m^{2}+x P_{z}^{2}\right)^{2}}-\frac{x}{|x-1| P_{z}^{2}\left[|x-1| P^{0}+(x-1) P^{z}\right]^{2}}\right\}, \\
& \tilde{q}_{b+c}^{R}\left(x, P^{z}\right)=\mathcal{Q}_{b+c}^{R}\left(x, n_{z}\right) \\
&=\frac{g_{s}^{2} C_{F}}{4 \pi}\left\{\frac{P^{0}|x-1|-P^{z}(x+1)}{P_{z}^{2}(x-1)|x-1|\left[P^{0}|x-1|+P^{z}(x-1)\right]}-\frac{P^{0} \sqrt{m^{2}+x^{2} P_{z}^{2}}+m^{2}-x P_{z}^{2}}{P^{z}(x-1) \sqrt{m^{2}+x^{2} P_{z}^{2}}\left(P^{0} \sqrt{m^{2}+x^{2} P_{z}^{2}}+m^{2}+x P_{z}^{2}\right)}\right\} \\
& \tilde{q}_{d}^{R}\left(x, P^{z}\right)=\mathcal{Q}_{d}^{R}\left(x, n_{z}\right)=\frac{g_{s}^{2} C_{F}}{4 \pi P_{z}^{2}|x-1|^{3}} .
\end{aligned}
$$

It is straightforward to check that, in the IMF, the one-loop corrections to the quasi-PDF do approach their light-cone counterparts, in a diagram-by-diagram basis.

Next we turn to the virtual corrections for the quasi-PDF. Each individual virtual one-loop diagram yields the following contribution, respectively,

$$
\begin{aligned}
\tilde{q}_{a}^{V}\left(x, P^{z}\right) & =\delta(x-1) \frac{g_{s}^{2} C_{F}}{2 \pi m^{2}}\left(\frac{P_{0}^{2}+P_{z}^{2}}{2 P_{z}^{2} \eta^{2}}-\frac{P_{0}^{2}+P_{z}^{2}}{P^{z} P^{0} \eta}+1\right) \\
\tilde{q}_{b+c}^{V}\left(x, P^{z}\right) & =Q_{b+c}^{V}\left(x, n_{z}\right)=-\delta(x-1) \frac{g_{s}^{2} C_{F}}{2 \pi m^{2}}\left[\frac{1}{\eta^{2}}-\frac{2 P^{0}}{\eta P^{z}}+\frac{m^{4} \tanh ^{-1}\left(\frac{P^{z}}{P^{0}}\right)+P^{0} P^{z}\left(m^{2}+P_{0}^{2}\right)}{P_{0}^{3} P^{z}}\right] \\
\tilde{q}_{d}^{V}\left(x, P^{z}\right) & =Q_{d}^{V}\left(x, n_{z}\right)=-\delta(x-1) \frac{g_{s}^{2} C_{F}}{4 \pi P_{z}^{2} \eta^{2}}
\end{aligned}
$$

where the quadratic IR singularity emerges in each diagram.

In computing the virtual corrections for quasi-PDFs, analogous to (92) in the light-cone case, we again utilize the momentum fraction $\eta$ as an IR regulator, imposed on the $k^{z}$ integration,

$$
\int_{0}^{P^{z}} d k^{z} \Rightarrow P^{z} \int_{0}^{1} d x \Theta(1-x-\eta),
$$

where $x=k^{z} / P^{z}$ and $\eta \rightarrow 0^{+}$.

Summing up $\tilde{q}_{a, b+c, d}^{R}$ and $\tilde{q}_{a, b+c, d}^{V}$, we obtain the complete one-loop corrections to the quasi-PDF,

$$
\begin{aligned}
\tilde{q}\left(x, P^{z}\right)= & \frac{g_{s}^{2} m^{2}}{4 \pi P^{z}}\left[\frac{1}{(x-1)\left(m^{2}+P_{z}^{2} x^{2}\right)^{3 / 2}}\right]_{+} \\
& +\frac{g_{s}^{2}}{2 \pi \eta P^{0} P^{z}} \delta(x-1),
\end{aligned}
$$

which is suppressed by at least one inverse power of $P^{z}$. Note the linear IR divergence is still present, but accompanied with a $\mathcal{O}\left(1 / P_{z}^{2}\right)$ suppression factor.

Comparing (94) with (98), we verify that, to the one-loop order, both the light-cone and quasi-PDFs do share the same IR behavior at the leading power in $1 / P^{z}$, which is simply zero.

Integrating $\tilde{q}\left(x, P^{z}\right)$ in (98) over the entire range of $x$ generates the one-loop correction to the vector current $\bar{\psi} \gamma^{z} \psi$. This nonvanishing integral indicates that the vector current no longer conserves, with the extent of violation of $\mathcal{O}\left(g_{s}^{2} / P_{z}^{2}\right)$, clearly a higher-twist effect. We suspect that the breaking of vector current conservation may originate from the fact that the momentum cutoff in the $k^{z}$ integration likely violates Lorentz invariance. To check this assumption, we also recalculate the one-loop correction to the vector current in DR, and confirm that the current conservation holds at one-loop order. Despite this nuisance, in order to be consistent with the rest of the paper, we will still stick to the soft momentum cutoff as a viable IR regulator.

In passing, it is worth mentioning that, in the matching between the light-cone and quasigeneralized parton distribution functions (GPDs) in four spacetime dimensions, a similar pattern has also been observed: for the $E$-type GPD, the light-cone and quasi-GPD differ in IR at one-loop order only by a higher-twist term [72,73].

As anticipated, due to UV finiteness of $\mathrm{QCD}_{2}$ at the oneloop level, the matching between the quasi-PDF and lightcone PDF turns out to be trivial, at least to this perturbative order, thereby the corresponding $Z$ factor is simply $\delta(\xi-1)$.

It is also illuminating to trace the origin of the $1 / P^{z_{-}}$ suppressed scaling behavior of quasi-PDF (hence the vanishing light-cone PDF) from another angle, i.e., from the time-ordered perturbation theory (TOPT), or often referred to as the old-fashioned perturbation theory. From (34), one can split the axial-gauge $\mathrm{QCD}_{2}$ Hamiltonian into the free and the interaction parts: 


$$
\begin{aligned}
H & =H_{0}+H_{\mathrm{int}}, \\
H_{0} & =\int d z \psi^{\dagger}(z)\left(-i \gamma^{5} \partial_{z}+m \gamma^{0}\right) \psi(z), \\
H_{\mathrm{int}} & =\iint d z d z^{\prime} \mathcal{H}_{\mathrm{int}}\left(z, z^{\prime}\right)=-\frac{g_{s}^{2}}{2} \sum_{a} \int d z d z^{\prime} \psi^{\dagger}(z) T^{a} \psi(z) \tilde{G}_{\rho}^{(2)}\left(z-z^{\prime}\right) \psi^{\dagger}\left(z^{\prime}\right) T^{a} \psi\left(z^{\prime}\right) .
\end{aligned}
$$

It is convenient to conduct the TOPT calculation for the partonic quasi distributions in the $A^{z}=0$ gauge, where the gauge links $\mathcal{W}$ in the quasi-PDF in (71) and quasi-DA in (81) simply disappear. Through the second order in $g_{s}$, it turns out that the quasi-PDF in (71) and quasi-DA in (81) can be recast into the equivalent TOPT format, each of which consists of two distinct time-ordering between $\bar{\psi} \gamma^{z} \psi$ and $H_{\text {int }}$ :

$$
\begin{aligned}
\tilde{q}\left(x, P^{z}\right)= & \iint d z_{1} d z_{2} \int \frac{d z}{4 \pi} e^{i x P^{z} z}\left\{\left\langle P\left|\bar{\psi}(z) \gamma^{z} \psi(0) \frac{1}{P^{0}-H_{0}} \mathcal{H}_{\text {int }}\left(z_{1}, z_{2}\right)\right| P\right\rangle_{C}+\left\langle P\left|\mathcal{H}_{\text {int }}\left(z_{1}, z_{2}\right) \frac{1}{P^{0}-H_{0}} \bar{\psi}(z) \gamma^{z} \psi(0)\right| P\right\rangle_{C}\right\}, \\
\tilde{\Phi}\left(x, P^{z}\right)= & \iint d z_{1} d z_{2} \int \frac{d z}{2 \pi} e^{i\left(x-\frac{1}{2}\right) P^{z} z}\left\{\left\langle p, P-p\left|\bar{\psi}\left(\frac{z}{2}\right) \gamma^{z} \psi\left(-\frac{z}{2}\right) \frac{1}{P^{0}-H_{0}} \mathcal{H}_{\text {int }}\left(z_{1}, z_{2}\right)\right| 0\right\rangle_{C}\right. \\
& \left.+\left\langle p, P-p\left|\mathcal{H}_{\text {int }}\left(z_{1}, z_{2}\right) \frac{1}{P^{0}-H_{0}} \bar{\psi}\left(\frac{z}{2}\right) \gamma^{z} \psi\left(-\frac{z}{2}\right)\right| 0\right\rangle_{C}\right\}
\end{aligned}
$$

where $H_{0}$ appearing in the energy denominator refers to the free part of the Hamiltonian, and $\mathcal{H}_{\text {int }}$ represents the instantaneous Coulomb interaction, both of which are defined in (99).

As before, we first replace the external hadronic states in (100a) by an on-shell quark with 2-momentum $P^{\mu}=\left(P^{0}, P^{z}\right)$. We proceed by inserting a complete set of eigenstates of $H_{0}$ immediately left to $1 /\left(P^{0}-H_{0}\right)$ in (100a). To obtain a nonvanishing result, the viable intermediate states are inevitably composed of three free particles, $q \bar{q} q$, which turns out to contribute to the real corrections for the quasi-PDF. ${ }^{13}$ We then compute the matrix elements of $\bar{\psi} \gamma^{z} \psi$ and $H_{\text {int }}$ separately, by contracting the field operators with the external partonic states in all possible way. Integrating over the spatial variables $z_{1}, z_{2}$ and $z$, we then end with the product of several momentumconserving $\delta$-function. One finally can write down all the order- $g_{s}^{2}$ contributions to the quasi-PDF. Each individual contribution corresponds to a particular way of contracting field operators and external states, which is schematically represented by the those TOPT diagrams in Fig. 6 .

Since the intermediate states must contain three particles for quasi-PDF, it is inevitable for the vacuum creation and annihilation vertices to arise in the TOPT diagrams, as can be clearly seen from the top row of Fig. 6. Obviously, it is

\footnotetext{
${ }^{13}$ If the intermediate states only consist of the single quark $q$, the matrix elements in (100a) then correspond to the virtual correction to the quasi-PDF. For simplicity, we will not bother to consider this piece of contribution.
}

the resulting large energy denominator that is responsible for the $1 / P^{z}$-suppressed behavior [74] of quasi-PDF. The quasi-PDF eventually vanishes when viewed in the IMF, which amounts to the vanishing light-cone PDF.

\section{B. LCDA and quasi-DA to one-loop order}

To access the LCDA and quasi-DA in perturbation theory, we proceed to replace a meson by a color-singlet $q \bar{q}$ pair. To justify perturbative expansion, in this subsection, we assume the weak coupling limit, $g_{s} \ll m$, has been taken. The corresponding one-loop diagrams for DAs are shown in Fig. 7.

In four spacetime dimensions, the one-loop matching factor linking the LCDA and quasi-DA, is more involved than the one linking the light-cone PDF and quasi-PDF. One needs to start with a more general momentum configuration $k^{+}=x P^{+}$and $p^{+}=y P^{+}$. Thus the matching factor $Z$ cannot be written as a single-variable function [72], instead must depend on both $x$ and $y$. Owing to the UV finiteness of $\mathrm{QCD}_{2}$, the matching factor is doomed to be trivial. Therefore, for illustrative purpose, we will focus on one specific kinematic configuration of the external "mesonic" state, $y=\frac{1}{2}$, that is, $p^{+}=\frac{p^{+}}{2}$, so that the $q$ and $\bar{q}$ equally partition the fictitious meson's total momentum. As a consequence, the DA becomes the function of $x$ only.

Following basically the same strategy as adopted in the one-loop calculation for the light-cone and quasiquark PDF, as described in Sec. VIII A, we obtain the one-loop corrections to the LCDA, $\Phi(x)$, and the quasi-DA, $\tilde{\Phi}\left(x, P^{z}\right)$ : 


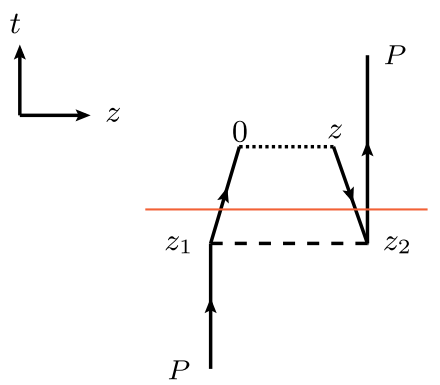

(a)

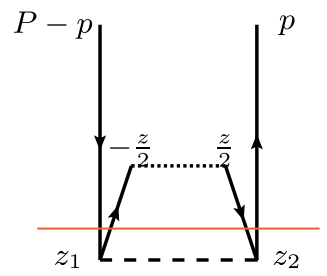

(e)

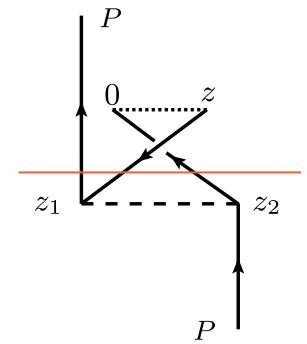

(b)

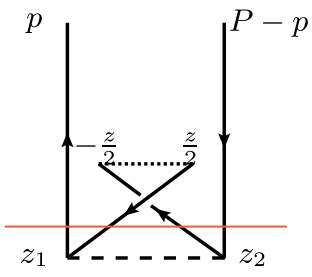

(f)

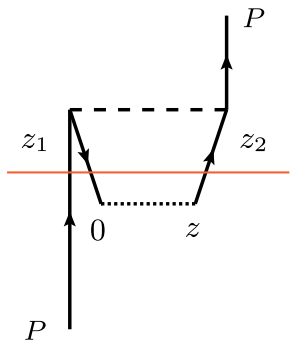

(c)

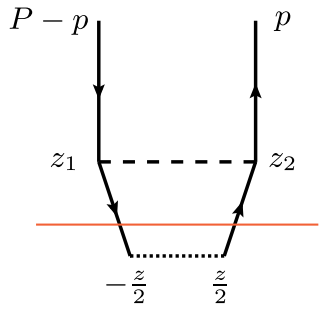

(g)

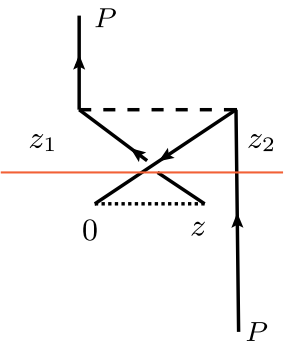

(d)

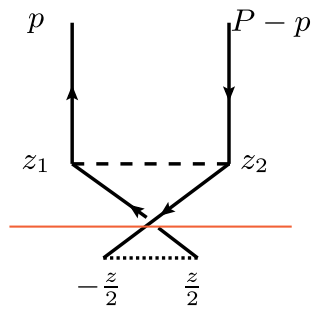

(h)

FIG. 6. Various TOPT diagrams that are responsible for the $\mathcal{O}\left(g_{s}^{2}\right)$ real correction contributions to quasi-PDF (a-d) and quasi-DA (eh). The dashed line represents the instantaneous color Coulomb potential. The horizontal (red) solid line specifies the allowed intermediate on-shell partonic states.
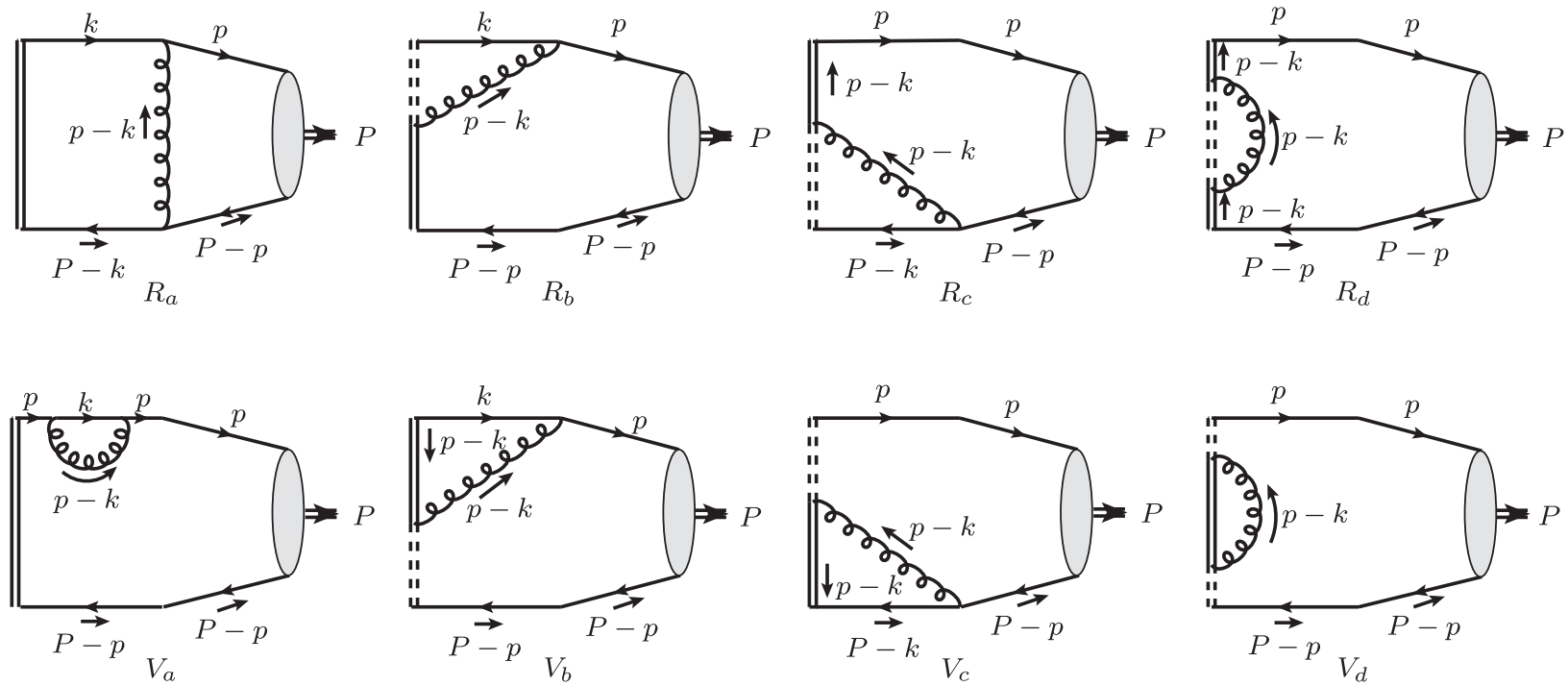

FIG. 7. One-loop Feynman diagrams for the real (upper row) and virtual (lower row) corrections to DA of a fictitious meson with momentum $P$, the Feynman diagrams show the one-loop corrections to the amplitude of extracting a quark (antiquark) with momentum $k(P-k)$ from a quark antiquark pair with momentum $p(P-p)$ correspondingly. For simplicity, we have omitted the wave function renormalization diagram for the antiquark line. Identical to the PDF case, the virtual corrections to the DA take the form of $Z_{F} \delta\left(x-\frac{1}{2}\right)$. The double line represents the gauge link in DA and quasi-DA definition (76) and (81), while the dashed double line represents the gauge link on which no net momentum flows.

$$
\begin{aligned}
\Phi(x) & = \begin{cases}-\left[\frac{4 g_{s}^{2} C_{F} x(1-x)}{\pi m^{2}(1-2 x)^{4}}\right]_{4+}+\frac{g_{s}^{2} C_{F}(1-4 \eta)}{16 \pi m^{2} \eta} \delta^{\prime \prime}\left(x-\frac{1}{2}\right) & 0<x<1 \\
0 & \text { Otherwise }\end{cases} \\
\tilde{\Phi}\left(x, P^{z}\right) & =\left\{\frac{g_{s}^{2} C_{F}}{\pi(1-2 x)^{2} P^{z}}\left[-\frac{2 P^{0}\left(m^{2}+x(1-x) P_{z}^{2}\right)}{m^{2}(1-2 x)^{2} P_{z}^{2}}+\frac{1}{\sqrt{m^{2}+(1-x)^{2} P_{z}^{2}}} \frac{x(x-1) P_{z}^{2}-x P^{0} \sqrt{m^{2}+(x-1)^{2} P_{z}^{2}}+2 m^{2}(x-1)}{-P^{0} \sqrt{m^{2}+(x-1)^{2} P_{z}^{2}}+2 m^{2}-x P_{z}^{2}+P_{z}^{2}}\right]\right. \\
& +(x \rightarrow 1-x)\}_{4+}-\frac{g_{s}^{2} C_{F}}{16 \pi m^{2}\left(P^{z}\right)^{3}} \delta^{\prime \prime}\left(x-\frac{1}{2}\right)\left[4 P^{z}\left(P_{0}^{2}-m^{2}\right)-\frac{P_{0}^{3}}{\eta}\right],
\end{aligned}
$$


where a "4-plus" prescription has been introduced. This prescription is understood in a distributive sense, which is defined as

$$
\int_{x_{1}}^{x_{2}} d x\left[\frac{g(x)}{\left|x-\frac{1}{2}\right|^{4}}\right]_{4+} f(x)=\int_{0}^{1} d x \frac{g(x)}{\left|x-\frac{1}{2}\right|^{4}}\left[f(x)-f\left(\frac{1}{2}\right)-f^{\prime}\left(\frac{1}{2}\right)\left(x-\frac{1}{2}\right)-\frac{f^{\prime \prime}\left(\frac{1}{2}\right)}{2 !}\left(x-\frac{1}{2}\right)^{2}-\frac{f^{\prime \prime \prime}\left(\frac{1}{2}\right)}{3 !}\left(x-\frac{1}{2}\right)^{3}\right],
$$

where $f(x)$ is any smooth functions that are regular at $x=\frac{1}{2}$. We further assume $g(x)$ is symmetric under the exchange $x \leftrightarrow 1-x$, so that $g^{\prime}\left(\frac{1}{2}\right)=0$. The integration boundaries are $x_{1}=0, x_{2}=1$ for LCDA, and $x_{1}=-x_{2}=$ $-\infty$ for quasi-DA, respectively. In (101a) and (101b), we have employed some distribution identities to express the DAs in terms of these "4-plus" distributions. More details about those identities can be found in Appendix B.

In contrast to (94) and (98) in the PDF case, one sees that, to the one-loop order, the quasi-DA contains some leading-twist pieces that are not suppressed by powers of $1 / P^{z}$. As anticipated, boosting (101b) to IMF, one readily recovers (101a). The difference between quasi-DA and LCDA is certainly of the higher-twist origin, of the order $g_{s}^{2} / P_{z}^{2}$.

Examining (101a) and (101b), reassuringly, we do observe that both LCDA and quasi-DA possess the identical linear IR singularity, $\propto \frac{g_{s}^{2}}{m^{2} \eta} \delta^{\prime \prime}\left(x-\frac{1}{2}\right)$.

It is again elucidating to see why the quark DA, in contrast to the quark PDF, contains a leading twist term, from the angle of time-ordered perturbation theory. Similar to what is done to quasi-PDF, we also insert a complete set of eigenstates of $H_{0}$ immediately left to $1 /\left(P^{0}-H_{0}\right)$ in (100b). Unlike the case of quasi-PDF, here the allowed intermediate states can be either $q \bar{q}$ or $q \bar{q} q \bar{q}$, in order to obtaina nonvanishing results for the real corrections to quasi-DA. Computing both matrix elements involving $\bar{\psi} \gamma^{z} \psi$ and $H_{\text {int }}$, exhausting all possible contractions between Dirac field operators and the external partonic states, integrating over the spatial variables $z_{1}, z_{2}$ and $z$, we finally end up with all the order- $g_{s}^{2}$ contributions to the quasi-DA. Each contribution specifies a particular way of contracting field operators and external states, which are represented by the those TOPT diagrams in lower row of Fig. 6.

In contrast to the case for quasi-PDF, apart from tetraquark states, the $q \bar{q}$ two-particle states also constitute the legitimate intermediate states. As a result, the corresponding TOPT diagrams, e.g. Fig. 6(c,d), in the lower row, are absent of the vacuum creation and annihilation vertices, therefore freed from suppression by large energy denominator. Consequently, the leading scaling behavior of the quasi-DA in the large momentum limit is $g_{s}^{2} /\left(m^{2} P_{z}^{0}\right)$, which leads to a nonvanishing LCDA when viewed in the IMF.

We now conclude this section. By explicitly working out the one-loop corrections to quark PDF and DA in
$\mathrm{QCD}_{2}$, we have firmly established the validity of the cornerstone of LaMET, viz., the partonic quasi- and light-cone distributions do share the identical IR behavior a t the leading power in $1 / P^{z}$. The one-loop correction to the DA appears to constitute a more nontrivial example than the PDF.

\section{SUMMARY AND OUTLOOK}

In this paper, we have carried out a comprehensive study of two important classes of meson parton distributions, the $\mathrm{PDF}$ and DA, in the context of the large- $N$ limit of $\mathrm{QCD}_{2}$. Our approach is entirely based upon the first principles of QCD. We have applied the Hamiltonian operator method as well as bosonization technique to construct both light-cone and quasidistributions out of the basic building blocks, that is, the 't Hooft wave function for the former, Bars-Green wave functions and the Bogoliubov angle for the latter. In a sense, equations (74) and (82) are the key formulae of this work. Unlike their fourdimensional counterparts, which can only be accessed by numerical lattice simulation in Euclidean spacetime, we have directly probed the quasidistributions in Minkowski spacetime, and have developed a thorough understanding about what they are made of in the twodimensional case.

We justify the 't Hooft wave function as the valid lightcone Fock state wave function of the hadron. Consequently, the quark PDF and LCDA can be directly built out of 't Hooft wave function, in an exceedingly simple manner. On the contrary, in the equal-time quantization, a pair of BarsGreen wave functions alone is not sufficient to express the quasidistributions, and one must supplement another important ingredient, the Bogoliubov-chiral angle, which may be viewed as characterizing the nonperturbative nature of the vacuum.

We have presented a comparative numerical study between light-cone PDFs and quasi-PDFs, as well as between LCDAs and quasi-DAs, for a variety of meson species. It is straightforward to see from (74) and (82) that, the quasidistributions do converge to their light-cone counterparts in the IMF. We also numerically verified the tendency that, the quasidistributions do approach their lightcone counterparts, when the meson gets more and more boosted. We have also observed an interesting pattern, that light meson's quasidistributions, in general, approach the light-cone distributions at a slower rate compared with the heavy mesons under boost. This somewhat counterintuive 
pattern is qualitatively consistent what is observed in lattice simulations in realistic four-dimensional QCD $[18,75]$.

Within the realm of perturbation theory, we have also investigated the one-loop corrections to the light-cone and quasidistributions in $\mathrm{QCD}_{2}$, yet abandoning the large- $N$ limit. We have verified the backbone of LaMET in this novel theoretical setting, that the IR behaviors of quasiand light-cone distributions are identical at the leading power in $1 / P^{z}$. It is theoretically interesting, since $\mathrm{QCD}_{2}$ has more severe IR divergence than $\mathrm{QCD}_{4}$. We do witness how the linear IR divergences in LCDA and quasi-DA agree with each in $\mathrm{QCD}_{2}$. Nevertheless, since $\mathrm{QCD}_{2}$ is a super-renormalizable theory, the matching $Z$ factor linking the light-cone with quasidistributions turns out to be trivial.

Equipped with the bosonization method, we are capable of computing virtually all the nonperturbative gaugeinvariant matrix elements in the 't Hooft model. For instance, besides quasi-PDFs, we are also able to compute the lattice cross section [39] as well as the pseudo- PDF $[37,38]$, which have been advocated as viable competitors of the quasi-PDF, presumed to be more efficient to extract the light-cone PDF. There is no principle difficulty in performing a similar study for these alternative options of parton distributions as in this work. To some extent, $\mathrm{QCD}_{2}$ may be viewed as an ideal and fruitful theoretical laboratory, which can examine many interesting ideas concerning a variety of parton distributions.

\section{ACKNOWLEDGMENTS}

We thank LiuJi Li for participating in the early stages of this work. We are grateful to Xiangdong Ji and Jianhui Zhang for useful discussions. The work of Y. J., S.-R. L., and R. Y. is supported, in part, by the National Natural Science Foundation of China under Grants No. 11475188 and No. 11621131001 (CRC110 by DFG and NSFC), by the IHEP Innovation Grant under Contract No. Y4545170Y2, and by the State Key Lab for Electronics and Particle Detectors. The work of X.-N.X. is supported by the Deutsche Forschungsgemeinschaft (Sino-German CRC 110).

\section{APPENDIX A: ALTERNATIVE DEFINITIONS OF QUASI-PDF AND -DA: $\gamma^{z}$ Versus $\gamma^{0}$}

As mentioned in Sec. VB, one is free to invent different operator definitions for quasidistributions, all of which are legitimate provided that they can reduce to the correct light-cone distributions in IMF. It is said that they then form a universality class [67]. The difference among them must be suppressed by powers of $1 / P^{z}$.

In this Appendix, we wish to critically compare two simplest definitions for quasi-PDF:

$$
\begin{aligned}
\tilde{q}_{\gamma^{z}}(x, P)= & \int_{-\infty}^{+\infty} \frac{d z}{4 \pi} e^{-i x P^{z} z} \\
& \times\left\langle P_{n}^{0}, P\left|\bar{\psi}(z) \gamma^{z} \mathcal{W}[z, 0] \psi(0)\right| P_{n}^{0}, P\right\rangle_{C}, \\
\tilde{q}_{\gamma^{0}}(x, P)= & \int_{-\infty}^{+\infty} \frac{d z}{4 \pi} e^{-i x P^{z} z} \\
& \times\left\langle P_{n}^{0}, P\left|\bar{\psi}(z) \gamma^{0} \mathcal{W}[z, 0] \psi(0)\right| P_{n}^{0}, P\right\rangle_{C} .
\end{aligned}
$$

The first canonical definition follows from (71), which has already been investigated in the main text. The second definition is new, which we are going to explore. The subscript " $C$ " again implies that only the connected part of the matrix element is retained.

Through the operator approach and the Bogoliubov transformation, the functional forms of two different definitions of quasi-PDFs in terms of the $\tilde{\varphi}_{ \pm}(x, P)$ and $\theta$ angle can be worked out,

$$
\begin{aligned}
\tilde{q}_{n, \gamma^{2}}(x, P)= & \frac{P_{n}^{0}}{P} \sin \theta(x P)\left[\left(\varphi_{+}^{n}(x P, P)\right)^{2}+\left(\varphi_{-}^{n}(x P, P)\right)^{2}\right. \\
& \left.+\left(\varphi_{+}^{n}(-x P, P)\right)^{2}+\left(\varphi_{-}^{n}(-x P, P)\right)^{2}\right], \\
\tilde{q}_{n, \gamma^{0}}(x, P)= & \frac{P_{n}^{0}}{P}\left[\left(\varphi_{+}^{n}(x P, P)\right)^{2}-\left(\varphi_{-}^{n}(x P, P)\right)^{2}\right. \\
& \left.+\left(\varphi_{-}^{n}(-x P, P)\right)^{2}-\left(\varphi_{+}^{n}(-x P, P)\right)^{2}\right] .
\end{aligned}
$$

Here, (A2) simply duplicates (74). Absence of the factor $\sin \theta$ in (A2b) may account for why the new quasi-PDF approaches the light-cone PDF at a faster pace than the canonical one. We are curious to know whether this has any connection to the realistic $\mathrm{QCD}_{4}$.

In Fig. 8, we juxtapose two versions of quasi-PDFs viewed from different reference frames, for the four different specifies of ground-state mesons. From the plots, we clearly see the tendency that both versions of quasi-PDFs would converge to the corresponding light-cone PDF in IMF. However, they evolve quite differently under the Lorentz boost. When the meson momentum is small, $\tilde{q}_{\gamma^{0}}$ appears to converge at a considerably slower pace than $\tilde{q}_{\gamma^{z}}$; nevertheless, when the meson momentum gets large, $\tilde{q}_{\gamma^{0}}$ appears to converge faster than $\tilde{q}_{\gamma^{z}}$. If this pattern persists in $\mathrm{QCD}_{4}$, one may be persuaded that $\tilde{q}_{\gamma^{0}}$ is perhaps a more favorable choice for lattice simulation than $\tilde{q}_{\gamma^{z}}$.

From Fig. 8, one can also observe that how the evolution patterns of two different quasi-PDFs depend on the quark mass. For each meson species, one might be interested in the critical threshold point of the momentum-to-mass ratio, $r_{\text {crt }}=P_{\text {crt }}^{z} / M$, after which the $\tilde{q}_{\gamma^{0}}$ starts to have a better convergence behavior than $\tilde{q}_{\gamma^{z}}$. For lighter mesons $\left(\pi_{\chi}, \pi\right)$, the critical $r$ values are quite large, $r_{\text {crt }}^{\pi_{\chi}}, r_{\text {crt }}^{\pi}$ are about 5 . In contrast, for heavier mesons $(s \bar{s}, c \bar{c})$, the critical $r$ values are rather small, $r_{\mathrm{crt}}^{s \bar{s}} \approx 0.2$, and $r_{\mathrm{crt}}^{c \bar{c}} \approx 0.025$.

Next, we turn to the quasi-DAs. Like the quasi-PDF case, we also intend to compare two simplest definitions for quasi-DAs: 

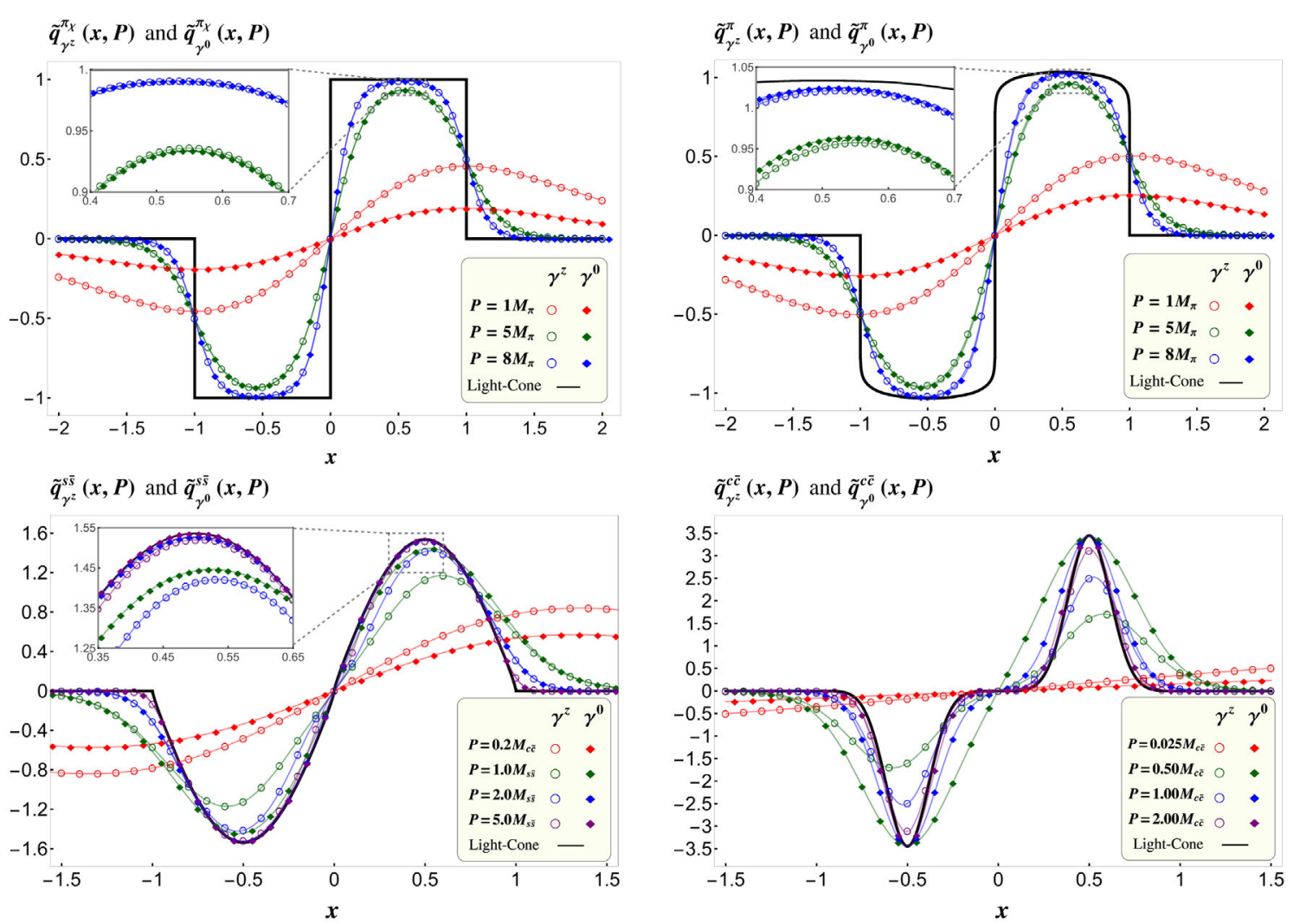

FIG. 8. Comparison between two versions of the quark quasi-PDF defined with $\gamma^{z}$ (open circles) and $\gamma^{0}$ (filled diamond), for four different ground-state mesons as specified in Table I.

$$
\begin{aligned}
& \tilde{\Phi}_{2 n, \gamma^{z}}(x, P)=\frac{1}{f^{(2 n)}} \int_{-\infty}^{+\infty} \frac{d z}{2 \pi} e^{i\left(x-\frac{1}{2}\right) P z}\left\langle P_{2 n}^{0}, P\left|\bar{\psi}\left(\frac{z}{2}\right) \mathcal{W}\left[\frac{z}{2},-\frac{z}{2}\right] \gamma^{z} \gamma_{5} \psi\left(-\frac{z}{2}\right)\right| \Omega\right\rangle, \\
& \tilde{\Phi}_{2 n, \gamma^{0}}(x, P)=\frac{1}{f^{(2 n)}} \int_{-\infty}^{+\infty} \frac{d z}{2 \pi} e^{i\left(x-\frac{1}{2}\right) P z}\left\langle P_{2 n}^{0}, P\left|\bar{\psi}\left(\frac{z}{2}\right) \mathcal{W}\left[\frac{z}{2},-\frac{z}{2}\right] \gamma^{0} \gamma_{5} \psi\left(-\frac{z}{2}\right)\right| \Omega\right\rangle,
\end{aligned}
$$

Utilizing the operator approach together with the Bogoliubov transformation, these two different versions of quasi-DA can be expressed as

$$
\begin{aligned}
& \tilde{\Phi}_{2 n, \gamma^{z}}(x, P)=\frac{1}{f^{(2 n)}} \sqrt{\frac{N}{\pi}} \sqrt{\frac{P^{0}}{P}} \sin \frac{\theta(x P)+\theta(P-x P)}{2}\left[\varphi_{+}^{2 n}(x P, P)+\varphi_{-}^{2 n}(x P, P)\right], \\
& \tilde{\Phi}_{2 n, \gamma^{0}}(x, P)=\frac{1}{f^{(2 n)}} \sqrt{\frac{N}{\pi}} \sqrt{\frac{P^{0}}{P}} \cos \frac{\theta(x P)-\theta(P-x P)}{2}\left[\varphi_{+}^{2 n}(x P, P)-\varphi_{-}^{2 n}(x P, P)\right],
\end{aligned}
$$

where $f^{(2 n)}$ is the decay constant of the $2 n$th mesonic state, one of whose explicit expressions has been given in (83). From Eq. (3.10) of Ref. [40], one can find another equivalent expression of $f^{(2 n)}$, from which one immediately sees that $\tilde{\Phi}_{2 n, \gamma^{0}}$ in (A4b) also obeys the normalization condition (84).

For the canonical quasi-DA, we have actually duplicated (82) for (A4a). The new quasi-DA assumes the form of (A4b). One readily sees that both types of quasi-DAs approach the LCDA in the IMF, therefore they belong to the same universality class. Nevertheless, it is not straightforward to see which trigonometric function, $\sin [(\theta(x P)+$ $\theta(P-x P)) / 2]$ or $\cos [(\theta(x P)-\theta(P-x P)) / 2]$, approaches unity at a faster pace as $P \rightarrow \infty$. Therefore, just by inspection of the analytical form, it is difficult to judge which definition of quasi-DAs bears better convergence behavior. 

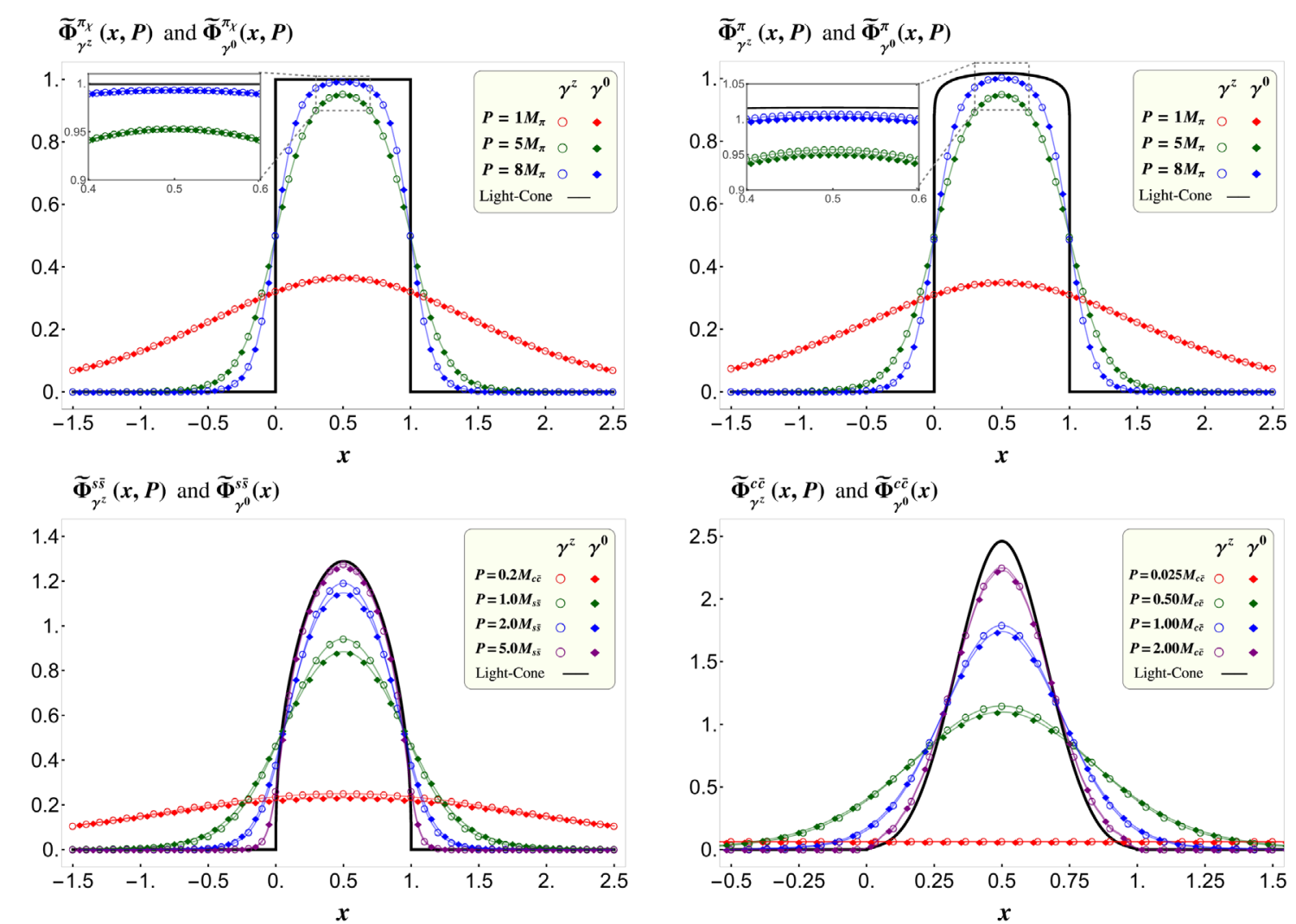

FIG. 9. Comparison between two versions of the quasi-DA defined with $\gamma^{z}$ (open circles) and $\gamma^{0}$ (filled diamond), for four different ground-state mesons as specified in Table I.

From Fig. 9, we see that two versions of quasi-DAs for the chiral pion are identical. This can be readily proved, since the analytical expressions for Bars-Green wave functions for $\pi_{\gamma}$ are exactly known [40,59]. For other massive mesons, it turns out that $\tilde{\Phi}_{\gamma^{z}}$ has always better convergence behavior than $\tilde{\Phi}_{\gamma^{0}}$, irrespective of the velocity of the boosted frame. The difference between these two quasi-DAs are always insignificant.

\section{APPENDIX B: DISTRIBUTION IDENTITIES ENCOUNTERED IN MOMENTUM CUTOFF IR REGULARIZATION}

In this Appendix, we collect some useful distribution identities that enable us to rewrite the LCDA and quasi-DA in terms of the "4-plus" distributions in Eq. (101). The validity of the following identities can be examined by picking up an arbitrary test function $f(x)$. Rather than specialize to the "4-plus" distribution, for the sake of generality, here we will introduce a " $n$-plus" distribution that would appear in a loop calculation implementing the IR momentum cutoff. For the light-cone loop integral, the " $n$-plus" distribution is defined as

$$
\begin{aligned}
& \int_{0}^{1} d x\left[\frac{g(x)}{\left|\frac{1}{2}-x\right|^{n}}\right]_{n+} f(x) \\
& \equiv \int_{0}^{1} d x \frac{g(x)}{\left|\frac{1}{2}-x\right|^{n}}\left[f(x)-\sum_{i=0}^{n} \frac{1}{i !} f^{(i)}\left(\frac{1}{2}\right)\left(x-\frac{1}{2}\right)^{i}\right] .
\end{aligned}
$$

For our purpose, we assume $g(x)$ is symmetric under the exchange $x \leftrightarrow 1-x$.

In Sec. VIII, we have adopted a soft momentum fraction $\eta$ as the IR regulator in the loop integration using light-cone coordinates [see (92)]. We often encounter the following type of integral:

$$
\begin{aligned}
\lim _{\eta \rightarrow 0} \int_{0}^{1} d x \Theta\left(\left|x-\frac{1}{2}\right|-\eta\right) \frac{g(x) f(x)}{\left|\frac{1}{2}-x\right|^{n}} \\
\quad=\int_{0}^{1} d x\left\{\left[\frac{g(x)}{\left|\frac{1}{2}-x\right|^{n}}\right]_{n+} f(x)+f(x) \sum_{i=0}^{\left[\frac{[n}{2}\right]} \frac{\delta^{(2 i)}\left(x-\frac{1}{2}\right)}{(2 i) !} \lim _{\eta \rightarrow 0} \int_{0}^{1} d y \Theta\left(\left|y-\frac{1}{2}\right|-\eta\right) \frac{g(y)\left|y-\frac{1}{2}\right|^{2 i}}{\left|y-\frac{1}{2}\right|^{n}}\right\} .
\end{aligned}
$$


In the second line, this integral has been rewritten in terms of the " $n$-plus" distribution together with a series of products of the $\delta$ function and some integrals in the $\eta \rightarrow 0$ limit. Since we only consider the DA of the flavor-neutral mesons, we have dropped the odd number of derivatives of the $\delta$ function, because those terms do not contribute when $g(x)$ is symmetric under $x \leftrightarrow 1-x$, since the odd number of derivatives of $g(x)$ vanishes at $x=1 / 2$.

The " $n$-plus" distribution in (B1) can be readapted to a convolution integral with unrestricted domain, $-\infty<$ $x<\infty$, which is relevant to the loop calculation for the quasi-DAs:

$$
\int_{-\infty}^{+\infty} d x\left[\frac{g(x)}{\left|\frac{1}{2}-x\right|^{n}}\right]_{n+} f(x)=\int_{-\infty}^{+\infty} d x \frac{g(x)}{\left|\frac{1}{2}-x\right|^{n}}\left[f(x)-\sum_{i=0}^{n} \frac{1}{i !} f^{(i)}\left(\frac{1}{2}\right)\left(x-\frac{1}{2}\right)^{i}\right]
$$

In Sec. VIII, we have also adopted a soft momentum fraction $\eta$ as the IR regulator in the loop integration using ordinary coordinates [see (97)]. We often confront the following type of integrals:

$$
\begin{aligned}
& \lim _{\eta \rightarrow 0} \int_{-\infty}^{+\infty} d x \Theta\left(\left|x-\frac{1}{2}\right|-\eta\right) \frac{g(x) f(x)}{\left|\frac{1}{2}-x\right|^{4}} \\
& \quad=\int_{-\infty}^{+\infty} d x\left\{\left[\frac{g(x)}{\left|\frac{1}{2}-x\right|^{n}}\right]_{n+} f(x)+f(x) \sum_{i=0}^{\left[\frac{n}{2}\right]} \frac{\delta^{(2 i)}\left(x-\frac{1}{2}\right)}{(2 i) !} \lim _{\eta \rightarrow 0} \int_{-\infty}^{+\infty} d y \Theta\left(\left|y-\frac{1}{2}\right|-\eta\right) \frac{g(y)\left|y-\frac{1}{2}\right|^{2 i}}{\left|y-\frac{1}{2}\right|^{n}}\right\}
\end{aligned}
$$

In the second line, we again have dropped the odd number of derivatives of the $\delta$ function. In this identity, it is necessary to assume that the test function $f(x)$ falls off sufficiently fast as $|x| \rightarrow \infty$.

[1] W. Detmold, W. Melnitchouk, and A.W. Thomas, Eur. Phys. J. Spec. Top. 3, 13 (2001).

[2] Ph. Hägler et al. (LHPC Collaboration), Phys. Rev. D 77, 094502 (2008).

[3] B. U. Musch, P. Hagler, M. Engelhardt, J. W. Negele, and A. Schafer, Phys. Rev. D 85, 094510 (2012).

[4] C. Alexandrou, M. Constantinou, S. Dinter, V. Drach, K. Hadjiyiannakou, K. Jansen, G. Koutsou, and A. Vaquero, J. High Energy Phys. 06 (2015) 068.

[5] V. M. Braun, S. Collins, M. Göckeler, P. Pérez-Rubio, A. Schäfer, R.W. Schiel, and A. Sternbeck, Proc. Sci., QCDEV2015 (2015) 009, arXiv:1510.07429.

[6] X. Ji, Phys. Rev. Lett. 110, 262002 (2013).

[7] X. Ji, Sci. China Phys. Mech. Astron. 57, 1407 (2014).

[8] X. Xiong, X. Ji, J. H. Zhang, and Y. Zhao, Phys. Rev. D 90, 014051 (2014).

[9] Y. Q. Ma and J. W. Qiu, arXiv:1404.6860.

[10] T. Ishikawa, Y. Q. Ma, J. W. Qiu, and S. Yoshida, Phys. Rev. D 96, 094019 (2017).

[11] X. Ji, J. H. Zhang, and Y. Zhao, Phys. Rev. Lett. 120, 112001 (2018).

[12] I. W. Stewart and Y. Zhao, Phys. Rev. D 97, 054512 (2018).

[13] C. Alexandrou, K. Cichy, M. Constantinou, K. Hadjiyiannakou, K. Jansen, H. Panagopoulos, and F. Steffens, Nucl. Phys. B923, 394 (2017).

[14] H. W. Lin, J. W. Chen, S. D. Cohen, and X. Ji, Phys. Rev. D 91, 054510 (2015).
[15] J. W. Chen, S. D. Cohen, X. Ji, H. W. Lin, and J. H. Zhang, Nucl. Phys. B911, 246 (2016).

[16] C. Alexandrou, K. Cichy, M. Constantinou, K. Hadjiyiannakou, K. Jansen, F. Steffens, and C. Wiese, Phys. Rev. D 96, 014513 (2017).

[17] C. Alexandrou, K. Cichy, V. Drach, E. Garcia-Ramos, K. Hadjiyiannakou, K. Jansen, F. Steffens, and C. Wiese, Phys. Rev. D 92, 014502 (2015).

[18] J. H. Zhang, J. W. Chen, X. Ji, L. Jin, and H. W. Lin, Phys. Rev. D 95, 094514 (2017).

[19] J. W. Chen, T. Ishikawa, L. Jin, H. W. Lin, Y. B. Yang, J. H. Zhang, and Y. Zhao, Phys. Rev. D 97, 014505 (2018).

[20] H. W. Lin, J. W. Chen, T. Ishikawa, and J. H. Zhang, arXiv:1708.05301.

[21] J. W. Chen, T. Ishikawa, L. Jin, H. W. Lin, A. Schäfer, Y. B. Yang, J. H. Zhang, and Y. Zhao, arXiv:1711.07858.

[22] J. W. Chen et al., arXiv:1712.10025.

[23] J. W. Chen, L. Jin, H. W. Lin, Y. S. Liu, Y. B. Yang, J. H. Zhang, and Y. Zhao, arXiv:1803.04393.

[24] T. Ishikawa, Y. Q. Ma, J. W. Qiu, and S. Yoshida, arXiv: 1609.02018.

[25] C.E. Carlson and M. Freid, Phys. Rev. D 95, 094504 (2017).

[26] X. Xiong, T. Luu, and U. G. Meißner, arXiv:1705.00246.

[27] J. W. Chen, X. Ji, and J. H. Zhang, Nucl. Phys. B915, 1 (2017).

[28] C. Monahan and K. Orginos, J. High Energy Phys. 03 (2017) 116. 
[29] C. Monahan, Phys. Rev. D 97, 054507 (2018).

[30] W. Wang, S. Zhao, and R. Zhu, Eur. Phys. J. C 78, 147 (2018).

[31] G. 't Hooft, Nucl. Phys. B72, 461 (1974).

[32] E. Witten, Nucl. Phys. B160, 57 (1979).

[33] S. R. Coleman, Aspects of Symmetry (Cambridge University Press, Cambridge, England, 1985), Chap. 8, 1/N.

[34] G. 't Hooft, Nucl. Phys. B75, 461 (1974).

[35] M. Burkardt, Phys. Rev. D 62, 094003 (2000).

[36] I. Bars and M. B. Green, Phys. Rev. D 17, 537 (1978).

[37] A. V. Radyushkin, Phys. Rev. D 96, 034025 (2017).

[38] K. Orginos, A. Radyushkin, J. Karpie, and S. Zafeiropoulos, Phys. Rev. D 96, 094503 (2017).

[39] Y. Q. Ma and J. W. Qiu, Phys. Rev. Lett. 120, 022003 (2018).

[40] Y. Jia, S. Liang, L. Li, and X. Xiong, J. High Energy Phys. 11 (2017) 151.

[41] A. R. Zhitnitsky, Phys. Lett. 165B, 405 (1985).

[42] K. Kikkawa, Ann. Phys. (N.Y.) 135, 222 (1981).

[43] A. Nakamura and K. Odaka, Phys. Lett. 105B, 392 (1981).

[44] S. G. Rajeev, Int. J. Mod. Phys. A 09, 5583 (1994).

[45] A. Dhar, G. Mandal, and S. R. Wadia, Phys. Lett. B 329, 15 (1994).

[46] A. Dhar, P. Lakdawala, G. Mandal, and S. R. Wadia, Int. J. Mod. Phys. A 10, 2189 (1995).

[47] M. Cavicchi, Int. J. Mod. Phys. A 10, 167 (1995).

[48] J. L. F. Barbon and K. Demeterfi, Nucl. Phys. B434, 109 (1995).

[49] K. Itakura, Phys. Rev. D 54, 2853 (1996).

[50] J. B. Kogut and D. E. Soper, Phys. Rev. D 1, 2901 (1970).

[51] C. G. Callan, Jr., N. Coote, and D. J. Gross, Phys. Rev. D 13, 1649 (1976).

[52] M. B. Einhorn, Phys. Rev. D 14, 3451 (1976).

[53] S. J. Brodsky, H. C. Pauli, and S. S. Pinsky, Phys. Rep. 301, 299 (1998).

[54] K. Hornbostel, Ph.D. thesis, Stanford Linear Accelerator Center, Stanford University, 1988.
[55] F. Lenz, M. Thies, K. Yazaki, and S. Levit, Ann. Phys. (N.Y.) 208, 1 (1991).

[56] S. Mandelstam, Nucl. Phys. B213, 149 (1983).

[57] G. Leibbrandt, Rev. Mod. Phys. 59, 1067 (1987).

[58] J. Hadamard, Lectures on Cauchy's Problem in Linear Partial Differential Equations (Dover, New York, 1923).

[59] Y. S. Kalashnikova and A. V. Nefediev, Usp. Fiz. Nauk 172, 377 (2002) [Phys. Usp. 45, 347 (2002)].

[60] M. E. Peskin and D. V. Schroeder, An Introduction to Quantum Field Theory (Addison-Wesley Publishing Company, Reading, 1997).

[61] M. Shifman, Advanced Topics in Quantum Field Theory: A Lecture Course (Cambridge University Press, Cambridge, England, 2012).

[62] M. Li, L. Wilets, and M. C. Birse, J. Phys. G 13, 915 (1987).

[63] F. Schwabl, Advanced Quantum Mechanics (Springer, New York, 2005), 3rd ed. See Sec. 3.2.2, "Bogoliubov Theory of the Weakly Interacting Bose Gas."

[64] P. J. d. A. Bicudo and J. E. F. T. Ribeiro, Phys. Rev. D 42, 1611 (1990).

[65] J. C. Collins and D. E. Soper, Nucl. Phys. B194, 445 (1982).

[66] J. Collins, Foundations of Perturbative QCD (Cambridge University Press, Cambridge, England, 2013).

[67] Y. Hatta, X. Ji, and Y. Zhao, Phys. Rev. D 89, 085030 (2014).

[68] G. P. Lepage and S. J. Brodsky, Phys. Rev. D 22, 2157 (1980).

[69] A. V. Radyushkin, arXiv:hep-ph/0410276.

[70] M. Burkardt, Phys. Rev. D 53, 933 (1996).

[71] X. Ji and J. H. Zhang, Phys. Rev. D 92, 034006 (2015).

[72] X. Ji, A. Schäfer, X. Xiong, and J. H. Zhang, Phys. Rev. D 92, 014039 (2015).

[73] X. Xiong and J. H. Zhang, Phys. Rev. D 92, 054037 (2015).

[74] S. Weinberg, Phys. Rev. 150, 1313 (1966).

[75] J. W. Chen, L. Jin, H. W. Lin, Y. S. Liu, A. Schäfer, Y. B. Yang, J. H. Zhang, and Y. Zhao, arXiv:1804.01483. 